\title{
SPECTRAL ANALYSIS OF DISCRETE ELLIPTIC OPERATORS AND APPLICATIONS IN CONTROL THEORY
}

\author{
DAMIEN ALLONSIUS*, FRANCK BOYER ${ }^{\dagger}$, AND MORGAN MORANCEY*
}

\begin{abstract}
In this paper we propose an analysis of discrete spectral properties for a finite difference discretization of quite general 1D second-order self-adjoint elliptic operators. We particularly investigate some (uniform with respect to the discretization parameter) qualitative behavior of eigenfunctions and eigenvalues.

With those estimates we manage to obtain new results for the construction of bounded families of controls for semi-discrete parabolic PDEs, in particular for boundary controls of a coupled parabolic system with fewer controls than equations.
\end{abstract}

Key words. Discrete spectral analysis, Sturm-Liouville operator, control theory, moments method, systems of parabolic equations.

AMS subject classifications. 34L16 - 35K10 - 65M06 - 93B05 - $93 \mathrm{~B} 40$

\section{Introduction.}

1.1. General context. We consider the differential operator

$$
\mathcal{A}:=-\partial_{x}\left(\gamma(x) \partial_{x} \cdot\right)+q(x) \cdot
$$

defined on the unit interval $\Omega=(0,1)$. The real valued functions $\gamma$ and $q$ are given and satisfy, for some $s \geq 0$,

$$
q \in \mathcal{C}^{s}(\bar{\Omega}) \text { and } \gamma \in \mathcal{C}^{s+1}(\bar{\Omega}) \text {, with } \gamma_{\min }:=\inf _{\Omega} \gamma>0 .
$$

We shall sometimes need to see $\mathcal{A}$ as a self-adjoint unbounded operator in $L^{2}(\Omega)$ whose domain is $D(\mathcal{A})=H_{0}^{1}(\Omega) \cap H^{2}(\Omega)$. In this setting, it is well-known that there exists an orthonormal basis of $L^{2}(\Omega)$ made of eigenfunctions of $\mathcal{A}$ that we denote by $\left(\phi_{k}\right)_{k \geq 1}$, the associated (real) eigenvalues being denoted by $\left(\lambda_{k}\right)_{k \geq 1}$. Those eigenvalues are simple and we assume that they are sorted in increasing order.

For any subset $O \subset \Omega$ and any $u \in L^{2}(\Omega)$, we define the semi-norm

$$
\|u\|_{L^{2}(O)}:=\left(\int_{O}|u(x)|^{2} d x\right)^{\frac{1}{2}},
$$

and for any smooth function $u: \bar{\Omega} \rightarrow \mathbb{R}$, we set

$$
\partial_{r} u:=u^{\prime}(1), \text { and } \partial_{l} u:=-u^{\prime}(0),
$$

which are the normal derivatives of $u$ at both ends of the interval $\Omega$.

In this section, we recall some qualitative properties of the eigenelements $\left(\phi_{k}, \lambda_{k}\right)_{k}$ that we are interested in. Of course, there exist much more accurate descriptions of the asymptotic behavior of the eigenelements of such operators (see [19]) but we only deal here with the same level of accuracy as the one that we shall achieve in the discrete framework.

\footnotetext{
*Aix-Marseille Université, CNRS, Centrale Marseille, I2M, UMR 7373, 13453 Marseille, France (damien.allonsius@univ-amu.fr, morgan.morancey@univ-amu.fr).

†Institut de Mathématiques de Toulouse, UMR 5219, Université de Toulouse, CNRS, UPS IMT, F-31062 Toulouse Cedex 9, France (franck.boyer@math.univ-toulouse.fr).
} 
Throughout this paper $C$ denotes a positive number that may vary from line to line. If necessary, we add information about dependency on parameters $p_{1}, p_{2}, \ldots p_{k}$ in the following way : $C:=C\left(p_{1}, p_{2}, \ldots, p_{k}\right)$.

Theorem 1.1. Assume that $\left(\mathrm{H}_{0}\right)$ holds. There exists $C_{1}(q, \gamma)>0$, such that

1. For any $k \geq 1$,

$$
\lambda_{k+1}-\lambda_{k} \geq C_{1} k
$$

2. For any $k \geq 1$

$$
\left|\partial_{r} \phi_{k}\right| \geq C_{1} k, \text { and }\left|\partial_{l} \phi_{k}\right| \geq C_{1} k .
$$

3. For any non-empty open subset $\omega \subset \Omega$, there exists $C_{2}(q, \gamma, \omega)>0$ such that for any $k \geq 1$,

$$
\left\|\phi_{k}\right\|_{L^{2}(\omega)} \geq C_{2}
$$

REMARK 1.1. Considering $\gamma=1$ and $q=0$ one can easily check that the bounds given in Theorem 1.1 are sharp. Indeed, in this case, we know that $\lambda_{k}=k^{2} \pi^{2}$, $\phi_{k}(x)=\sqrt{2} \sin \left(\sqrt{\lambda_{k}} x\right)$. Thus,

$$
\lambda_{k+1}-\lambda_{k}=(2 k+1) \pi^{2} \text { and }\left|\partial_{b} \phi_{k}\right|=\sqrt{2 \lambda_{k}}=\sqrt{2} \pi k, \quad \forall k \geq 1, \forall b \in\{l, r\},
$$

and for $\omega=(a, b) \subset(0,1)$, we have

$$
\left\|\phi_{k}\right\|_{L^{2}(\omega)}^{2}=\int_{a}^{b} 2 \sin ^{2}(k \pi x) \mathrm{d} x \underset{k \rightarrow \infty}{\longrightarrow} b-a=|\omega| .
$$

REMARK 1.2. By using the min-max principle and the exact knowledge of the eigenvalues of the Laplace operator as recalled in the previous remark, we can show that the eigenvalues of $\mathcal{A}$ satisfy

$$
\gamma_{\min } \pi^{2} k^{2}-\|q\|_{L^{\infty}} \leq \lambda_{k} \leq\|\gamma\|_{L^{\infty}} \pi^{2} k^{2}+\|q\|_{L^{\infty}}, \quad \forall k \geq 1 .
$$

The first point in Theorem 1.1 implies in particular the so-called gap property for the eigenvalues, which means that there exists some $\rho>0$ such that $\lambda_{k+1}-\lambda_{k} \geq \rho$ for any $k$.

It appears that all those properties allow, by using the so-called moments method (see [13]), to prove some null-controllability results for the parabolic problem associated with $\mathcal{A}$ (either for a distributed control or for a boundary control) as well as for some coupled parabolic systems associated with $\mathcal{A}$ with fewer controls than components. This will be detailled in section 5.1.

The main goal of this paper is to study whether or not the three results given in Theorem 1.1 still hold for a discrete version $\mathcal{A}^{h}$ of the differential operator uniformly with respect to the discretization parameter $h>0$. As a consequence, we shall obtain new uniform controllability results for the associated semi-discrete parabolic equation and systems via the moments method adapted to the discrete setting.

1.2. Notations and discrete framework. For any two real numbers $\alpha, \beta$ we define

$$
\llbracket \alpha, \beta \rrbracket:=[\min (\alpha, \beta), \max (\alpha, \beta)] \cap \mathbb{N},
$$

to be the set of all the integers between $\alpha$ and $\beta$.

Let us recall here the standard notation for the finite difference discretization of the differential operator $\mathcal{A}$. We consider an ordered set of $N+2$ distinct points of $[0,1]$ denoted by $\left(x_{i}\right)_{i=0}^{N+1}$ and satisfying $x_{0}=0$ and $x_{N+1}=1$. Let $\left(x_{i+1 / 2}\right)_{i=0}^{N}$ be the dual 
mesh points defined by $x_{i+1 / 2}:=\frac{x_{i+1}+x_{i}}{2}$. We set $h_{i+1 / 2}:=x_{i+1}-x_{i}$ for $i \in \llbracket 0, N \rrbracket$ and $h_{i}:=x_{i+1 / 2}-x_{i-1 / 2}=\frac{h_{i+1 / 2}+h_{i-1 / 2}}{2}$, for $i \in \llbracket 1, N \rrbracket$. As usual, the mesh size is defined by $h:=\max _{i \in \llbracket 0, N \rrbracket} h_{i+1 / 2}$. We use the standard abuse of notation that consists in using the index $h$ to denote any mesh-dependent quantity even though it obviously not only depends on $h$ but also on the whole geometry of the mesh points. For such a given mesh we introduce

$$
\Theta_{h}:=\frac{h}{\min _{i \in \llbracket 0, N \rrbracket} h_{i+1 / 2}},
$$

which is a measure of the (non-)uniformity of the mesh. A family of meshes such that $\left(\Theta_{h}\right)_{h}$ is bounded, is called quasi-uniform.

For any open subset $O \subset \Omega$, we define the discrete $L^{2}(O)$ semi-norm on $\mathbb{R}^{N}$ by

$$
\|U\|_{L^{2}\left(O_{h}\right)}:=\left(\sum_{\substack{i \in \llbracket 1, N \rrbracket \\ x_{i} \in O}} h_{i}\left|u_{i}\right|^{2}\right)^{\frac{1}{2}}, \forall U=\left(u_{i}\right)_{i=1}^{N} \in \mathbb{R}^{N},
$$

and for a time-dependent function $t \in(0, T) \mapsto U(t) \in \mathbb{R}^{N}$ we set

$$
\|U\|_{L^{2}\left(O_{h}^{T}\right)}:=\left(\int_{0}^{T}\|U(t)\|_{L^{2}\left(O_{h}\right)}^{2} d t\right)^{\frac{1}{2}} .
$$

It is easily seen that $\|\cdot\|_{L^{2}\left(\Omega_{h}\right)}$ is actually a Euclidian norm on $\mathbb{R}^{N}$, whose inner product is denoted by $\langle\cdot, \cdot\rangle_{L^{2}\left(\Omega_{h}\right)}$.

We set $q_{i}:=q\left(x_{i}\right), \gamma_{i+1 / 2}:=\gamma\left(x_{i+1 / 2}\right)$ and we define the square matrix $\mathcal{A}^{h}$ of size $N$ by

$$
\left(\mathcal{A}^{h} U\right)_{j}:=-\frac{1}{h_{j}}\left(\gamma_{j+1 / 2} \frac{u_{j+1}-u_{j}}{h_{j+1 / 2}}-\gamma_{j-1 / 2} \frac{u_{j}-u_{j-1}}{h_{j-1 / 2}}\right)+q_{j} u_{j}, \forall j \in \llbracket 1, N \rrbracket
$$

for any discrete function $U=\left(u_{i}\right)_{i=1}^{N} \in \mathbb{R}^{N}$, and any $j \in \llbracket 1, N \rrbracket$, with the usual convention that $u_{0}=u_{N+1}=0$ to take into account homogeneous Dirichlet boundary conditions. By analogy with the continuous setting we define the normal derivative of a discrete function $U \in \mathbb{R}^{N}$, taking into account the Dirichlet boundary condition, as follows

$$
\partial_{r} U:=\frac{0-u_{N}}{h_{N+1 / 2}}, \text { and } \partial_{l} U:=-\frac{u_{1}-0}{h_{1 / 2}} .
$$

It is well-known that the discrete operator $\mathcal{A}^{h}$ is a second order accurate approximation of the differential operator $\mathcal{A}$ (see [5]). Moreover, $\mathcal{A}^{h}$ is self-adjoint in the space $\left(\mathbb{R}^{N},\langle\cdot, \cdot\rangle_{L^{2}\left(\Omega_{h}\right)}\right)$ so that there exists a (finite) orthonormal family $\left(\phi_{k}^{h}\right)_{k=1}^{N} \subset \mathbb{R}^{N}$ made of eigenvectors of $\mathcal{A}^{h}$ (also referred to as discrete eigenfunctions in this paper), and associated with eigenvalues denoted by $\Lambda^{h}:=\left(\lambda_{k}^{h}\right)_{k=1}^{N}$. In other words, we have the relations

$$
\mathcal{A}^{h} \phi_{k}^{h}=\lambda_{k}^{h} \phi_{k}^{h}, \text { and }\left\langle\phi_{k}^{h}, \phi_{j}^{h}\right\rangle_{L^{2}\left(\Omega_{h}\right)}=\delta_{k j}, \quad \forall k, j \in \llbracket 1, N \rrbracket .
$$

All those eigenvalues are simple and we assume that they are sorted in increasing order. 
1.3. Presentation of the results. The results of this paper are split into two parts. We first study spectral properties of the discrete operator $\mathcal{A}^{h}$ similar to the ones given in Theorem 1.1 of Section 3 in the continuous setting. To prepare this analysis we shall give the corresponding proofs at the continuous level in Section 2. In Section 4, we propose some numerical simulations that illustrate our theoretical results. In a second part of the paper (Section 5), we give our main applications in discrete controllability theory for system of parabolic equations.

To begin with, notice that all computations are explicit in the case of discrete Laplace operator on a uniform mesh.

REMARK 1.3 (discrete Laplace operator on uniform meshes). Let $h_{*}=1 /(N+1)$ and denote by $\mathcal{A}^{h_{*}}$ the discrete operator obtained on the uniform mesh of size $h_{*}$ with $\gamma=1$ and $q=0$ (which is nothing but the very usual discrete Laplace operator). Then, the eigenelements of $\mathcal{A}^{h_{*}}$ are explicitely given by

$$
\lambda_{k}^{h_{*}}=\frac{4}{h_{*}^{2}} \sin ^{2}\left(\frac{k \pi h_{*}}{2}\right), \quad\left(\phi_{k}^{h_{*}}\right)_{i}=\sqrt{2} \sin \left(k \pi i h_{*}\right), \forall i, k \in \llbracket 1, N \rrbracket .
$$

In particular, we have

$$
4 k^{2} \leq \lambda_{k}^{h_{*}} \leq \pi^{2} k^{2}, \quad \forall k \in \llbracket 1, N \rrbracket,
$$

and

$$
\lambda_{k}^{h_{*}} \leq \lambda_{N}^{h_{*}}=\frac{4}{h_{*}^{2}} \cos ^{2}\left(\frac{\pi}{2} h_{*}\right) \leq \frac{4}{h_{*}^{2}}-4, \quad \forall k \in \llbracket 1, N \rrbracket .
$$

Here we used the standard inequality $\cos ^{2}(t) \leq 1-\frac{4}{\pi^{2}} t^{2}$ for any $0 \leq t \leq 1$.

By using the formulas recalled in the remark above, one can easily obtain the following result.

Proposition 1.1. Let $h_{*}$ and $\mathcal{A}^{h_{*}}$ be as defined in Remark 1.3. The following properties hold:

- Gap property:

$$
12 \leq \min _{k \in \llbracket 1, N-1 \rrbracket}\left(\lambda_{k+1}^{h_{*}}-\lambda_{k}^{h_{*}}\right) \leq 3 \pi^{2},
$$

moreover the minimum is achieved for $k=1$ and $k=N-1$.

- Discrete normal derivative estimate: for $b \in\{l, r\}$

$$
\begin{gathered}
2 \sqrt{2} k \leq\left|\partial_{b} \phi_{k}^{h_{*}}\right| \leq \sqrt{2} k \pi, \quad \forall k \in \llbracket 1, N / 2 \rrbracket, \\
2 \sqrt{2}(N+1-k) \leq\left|\partial_{b} \phi_{k}^{h_{*}}\right| \leq \sqrt{2}(N+1-k) \pi, \quad \forall k \in \llbracket N / 2, N \rrbracket .
\end{gathered}
$$

- $L^{2}$-norm estimate: for any non empty open subset $\omega \subset \Omega$, there exists $h_{0}(\omega)>$ 0 and $a C(q, \gamma, \omega)$ such that, for any $h_{*}<h_{0}$, we have

$$
\left\|\phi_{k}^{h_{*}}\right\|_{L^{2}\left(\omega_{h}\right)} \geq C, \quad \forall k \in \llbracket 1, N \rrbracket .
$$

Some comments are in order. There are fundamental differences between Theorem 1.1 and Proposition 1.1. We first observe that the uniform gap property holds but not the stronger property 1 of Theorem 1.1 since the distance between the two largest discrete eigenvalues of $\mathcal{A}^{h_{*}}$ does not tend to infinity. Similarly, the discrete normal derivative of the eigenfunction $\phi_{N}^{h_{*}}$ for instance does not tend to infinity as $N \rightarrow \infty$ (and simultaneously $h_{*} \rightarrow 0$ of course). It thus already appears that the qualitative 


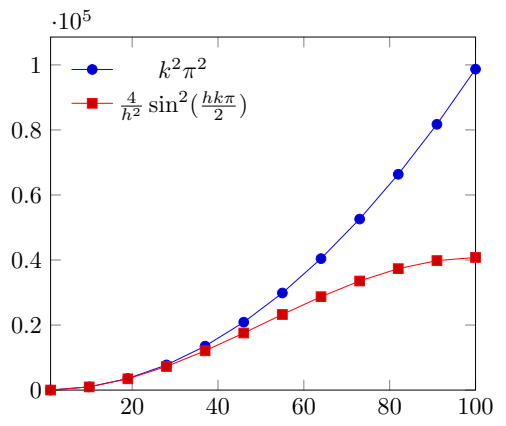

(a) Discrete and continuous eigenvalues $(N=100, k \in \llbracket 1, N \rrbracket)$.

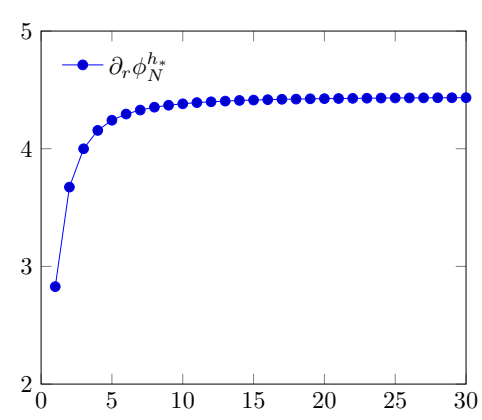

(b) $N \mapsto \partial_{r} \phi_{N}^{h_{*}}$.

Figure 1: Differences between discrete and continuous eigenelements.

behavior of the eigenelements of $\mathcal{A}^{h_{*}}$ for the upper part of the spectrum may be quite different from the one of the continuous operator, as we can see on Figure 1. However, for the lower part of the spectrum the properties are quite similar to the ones of $\mathcal{A}$.

Our aim is to generalize the three estimates of Proposition 1.1 to non uniform meshes and non constant coefficients operators for which we do not possess analytic formulas for the discrete eigenfunctions and eigenvalues.

REMARK 1.4 (Spectral properties for a given mesh and operator). It is worth noticing that, by straightforward linear algebra arguments, the following properties hold without any assumption on the mesh or on the coefficient of the operator

$$
\begin{gathered}
\min _{1 \leq k \leq N-1}\left(\lambda_{k+1}^{h}-\lambda_{k}^{h}\right)>0, \\
\min _{1 \leq k \leq N}\left|\partial_{b} \phi_{k}^{h}\right|>0, \forall b \in\{l, r\}, \\
\min _{1 \leq k \leq N}\left\|\phi_{k}^{h}\right\|_{L^{2}\left(\omega_{h}\right)}>0,
\end{gathered}
$$

as soon as $\omega$ contains at least two points of the mesh. However, those bounds from below will in general tend to 0 as $h \rightarrow 0$ (see the numerical experiments in Section 4). In that respect, the case of a constant coefficient operator on a uniform mesh is very particular (see Proposition 1.1).

Therefore, our main concern in this paper will be to obtain lower bounds for the quantities above that are uniform with respect to $h$. More precisely, we will try to identify the maximal subset of $\llbracket 1, N \rrbracket$ for which such uniform bounds hold.

REMARK 1.5. From the discussion above, it already appears that this problem cannot be tackled by classical numerical analysis arguments. Indeed, although it can be established that in the continous setting $\lambda_{k+1}-\lambda_{k} \geq C k$ (see Theorem 1.1, first estimate), we cannot deduce directly from this estimate that a gap property holds in the discrete setting since Figure 1 reveals that the error of approximation $\left|\lambda_{N}-\lambda_{N}^{h}\right|$ can be as large as $C N^{2}$, see also Proposition 3.1. However, for the low frequencies $\left(k \in\left\{1, \ldots, C N^{\alpha}\right\}\right.$ with a suitable choice of $\alpha<1$ and $C$ (see Theorem 3.1)), one can get that $\left|\lambda_{k}-\lambda_{k}^{h}\right| \leq \epsilon k$, with $\epsilon$ sufficiently small so that estimate 1 of Theorem 1.1 allows us to deduce a uniform gap estimate in the discrete setting for this particular 
portion of the spectrum. We will apply this argument in the most general setting we consider. Nevertheless, with stronger assumptions, we develop a strategy to obtain better estimates.

We will consider three slightly different sets of hypothesis for the regularity of the meshes and the coefficients of our operator, namely

quasi-uniform meshes, $q$ and $\gamma$ satisfying $\left(\mathrm{H}_{2}\right)$,

uniform meshes, $q$ and $\gamma$ satisfying $\left(\mathrm{H}_{0}\right)$,

uniform meshes, $q$ and $\gamma$ satisfying $\left(\mathrm{H}_{0}\right)$, and $\gamma$ is constant.

In the remark below we show that the results we shall obtain in Section 3 about operator $\mathcal{A}^{h}$ on uniform meshes in the setting (S2) still hold, in fact, for a wide class of regular but non uniform meshes. Yet, to simplify the presentation of the next sections, we shall always deal with uniform meshes in the setting (S2).

REMARK 1.6 (Families of regular meshes). In this remark, we use the notation $\mathcal{A}_{\gamma, q}^{h}$ for the discrete operator defined on any given mesh and for any couple of co-

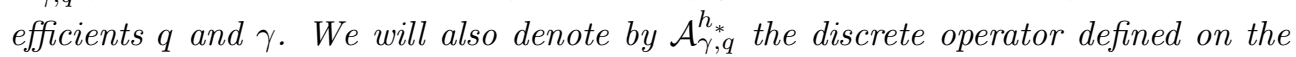
uniform mesh with the same number of cells and a mesh size $h_{*}=\frac{1}{N+1}$.

We define the vector $\alpha^{h}=\left(\alpha_{i}\right)_{1 \leq i \leq N}$ by

$$
\alpha_{i}:=\sqrt{\frac{h_{i}}{h_{*}}},
$$

and we set

$$
\bar{\gamma}_{i+1 / 2}:=\frac{h_{*}^{2}}{h_{i+1 / 2} \sqrt{h_{i} h_{i+1}}} \gamma_{i+1 / 2}, \forall i \in \llbracket 0, N \rrbracket,
$$

and

$$
\bar{q}_{i}:=q_{i}-\frac{1}{\alpha_{i}} \mathcal{A}_{\bar{\gamma}, 0}^{h_{*}} \alpha^{h}, \forall i \in \llbracket 1, N \rrbracket .
$$

With those notations, one can check by direct algebraic manipulations that, if $u^{h} \in \mathbb{R}^{N}$ and $\lambda \in \mathbb{R}$ satisfy $\mathcal{A}_{\gamma, q}^{h} u^{h}=\lambda u^{h}$, then we have

$$
\mathcal{A}_{\bar{\gamma}, \bar{q}}^{h_{*}}\left(\alpha^{h} u^{h}\right)=\lambda\left(\alpha^{h} u^{h}\right)
$$

In other words, the operators $\mathcal{A}_{\gamma, q}^{h}$ and $\mathcal{A}_{\bar{\gamma}, \bar{q}}^{h_{*}}$ have exactly the same eigenvalues and the corresponding eigenfunctions are deduced one from each other by a term by term multiplication by the coefficients $\alpha_{i}$.

It follows that, any (uniform with respect to $h$ ) spectral property proved on the modified operator $\mathcal{A}_{\bar{\gamma}, \bar{q}}^{h_{*}}$ on a uniform mesh, lead to the equivalent property for the original operator $\mathcal{A}_{\gamma, q}^{h}$ on a non-uniform grid. The price to pay in this manipulation lies in the fact that the new coefficients $\bar{\gamma}$ and $\bar{q}$ actually depend on the geometry of the initial mesh. In particular, one needs to control their "regularity" for uniform estimates to hold. More precisely, we need to make sure that they are, in some sense, bounded in $\mathcal{C}^{1}$ and $\mathcal{C}^{0}$ respectively (as required in the assumption $\left(\mathrm{H}_{0}\right)$ ). Of course, this has to be carefully defined since those discrete coefficients are not, a priori, obtained by sampling some functions on the primal and dual meshes. 
As an important example, it can be shown by using standard Taylor formulas that any mesh obtained as the image of a uniform mesh by a diffeomorphism $\vartheta:[0,1] \rightarrow$ $[0,1]$ of class $\mathcal{C}^{3}$ as follows

$$
x_{i}=\vartheta\left(i h_{*}\right), \quad \forall i \in \llbracket 1, N \rrbracket,
$$

fulfills all the required regularity properties for our results to hold.

Those particular meshes families were already considered for instance in [8] but also in the recent work [12].

Observe however that the same manipulation does not hold in the setting (S3) since it transforms a constant-diffusion problem into a variable and mesh-dependent diffusion problem.

1.3.1. Discrete spectral properties. Let us first gather here the various results we will prove on qualitative properties of the eigenelements of $\mathcal{A}^{h}$. Without loss of generality we will assume that $q \geq 0$ so that all the eigenvalues $\lambda_{k}^{h}$ and $\lambda_{k}$ are positive. Otherwise, one need to replace $\lambda_{k}^{h}$ by $\lambda_{k}^{h}+\|q\|_{\infty}$ in all the results below.

Properties of discrete eigenfunctions. Let $\omega$ be a fixed non empty open subdomain of $\Omega$.

- In the case (S1), we obtain (Theorem 3.1) bounds from below of the following kind

$$
\left\|\phi_{k}^{h}\right\|_{L^{2}\left(\omega_{h}\right)}^{2} \geq C_{1} e^{-C_{2} \sqrt{\lambda_{k}^{h}}}, \quad \forall k \in \llbracket 1, N \rrbracket,
$$

for any non empty open subdomain $\omega \subset \Omega$ and $h$ small enough, and

$$
\left|\partial_{b} \phi_{k}^{h}\right| \geq C_{1} \sqrt{\lambda_{k}^{h}} e^{-C_{2} \sqrt{\lambda_{k}^{h}}}, \quad \forall k \in \llbracket 1, N \rrbracket, \forall b \in\{l, r\},
$$

where $C_{1}$ and $C_{2}$ do not depend on the mesh size $h$.

- In the case (S2), we can refine those estimates on a constant lower fraction of the spectrum by proving (Theorem 3.2) that there exists a constant $0<\alpha \leq 1$ (depending only on $\gamma$ and $\omega$ ) such that

$$
\begin{gathered}
\left\|\phi_{k}^{h}\right\|_{L^{2}\left(\omega_{h}\right)}^{2} \geq C_{1}, \quad \forall k \in \llbracket 1, \alpha N \rrbracket \\
\left|\partial_{b} \phi_{k}^{h}\right| \geq C_{1} \sqrt{\lambda_{k}^{h}}, \quad \forall k \in \llbracket 1, \alpha N \rrbracket, \quad \forall b \in\{l, r\} .
\end{gathered}
$$

Moreover, we can give a sharp estimate of the best constant $\alpha$ for which those properties hold, as attested by numerical experiments in Section 4.

- Finally, in the case (S3), we can recover optimal (in a sense to be made precise later) estimates on the whole spectrum (Theorem 3.3)

$$
\begin{gathered}
\left\|\phi_{k}^{h}\right\|_{L^{2}\left(\omega_{h}\right)}^{2} \geq C_{1}\left(1-\frac{h^{2}\left(\lambda_{k}^{h}-\|q\|_{L^{\infty}}\right)}{4 \gamma}\right), \quad \forall k \in \llbracket 1, N \rrbracket, \\
\left|\partial_{b} \phi_{k}^{h}\right| \geq C_{1} \sqrt{\lambda_{k}^{h}} \sqrt{1-\frac{h^{2}\left(\lambda_{k}^{h}-\|q\|_{\left.L^{\infty}\right)}\right.}{4 \gamma}}, \quad \forall k \in \llbracket 1, N \rrbracket, \forall b \in\{l, r\} .
\end{gathered}
$$

Observe that in the settings (S2) and (S3), for a fixed value of $k$ and $h \rightarrow 0$, since it is well-known that $\lambda_{k}^{h} \rightarrow \lambda_{k}$, we recover the same asymptotic lower bounds as for the continuous operator $\mathcal{A}$. The latter are provided by Theorem 1.1 . 
From those estimates, we will deduce in particular that for some $C_{2}>0$,

$$
\begin{gathered}
\left\|\phi_{k}^{h}\right\|_{L^{2}\left(\omega_{h}\right)}^{2} \geq \frac{C_{2}}{\lambda_{k}^{h}}, \quad \forall k \in \llbracket 1, N \rrbracket, \\
\left|\partial_{b} \phi_{k}^{h}\right| \geq C_{2}, \quad \forall k \in \llbracket 1, N \rrbracket, \quad \forall b \in\{l, r\} .
\end{gathered}
$$

Gap property for the eigenvalues. We study here the so-called uniform gap property, that is : is there a constant $C>0$, independent of $h>0$, such that

$$
\lambda_{k+1}^{h}-\lambda_{k}^{h} \geq C, \forall k \in \llbracket 1, N-1 \rrbracket \quad ?
$$

In Proposition 1.1 we have shown that this property holds for the discrete Laplacian on a uniform mesh.

In the general cases where $q$ and $\gamma$ only satisfy $\left(\mathrm{H}_{0}\right)$, numerical experiments (Section 4) reveal that the uniform gap property may fail, at least on the upper part of the spectrum. More precisely, one can exhibit two distinct eigenvalues that are exponentially close as $h \rightarrow 0$ in the case where $\gamma$ is not a constant function.

Thus, a reasonnable question is: does a gap property hold if one only considers a lower fraction of the spectrum, or more precisely is there a positive increasing function $F: \mathbb{N} \rightarrow \mathbb{R}$ such that for some $C>0$ we have for any $h>0$,

$$
\lambda_{k+1}^{h}-\lambda_{k}^{h} \geq C, \forall k \in \llbracket 1, F(N) \rrbracket \quad ?
$$

In the case (S2), we prove existence of a sharp (according to numerical simulations) parameter $\alpha \in(0,1]$ such that $F(N)=\alpha N$ satisfies the above requirements. In the case (S3), we prove a weaker property, namely that for any $\beta>0$ and any mesh satisfying $\Theta_{h} \leq \beta$, there exists $\alpha:=\alpha(\gamma, q, \beta)>0$ such that $F(N)=\alpha N^{2 / 5}$.

1.3.2. Applications to the numerical approximation of control problems. As a main application of the previous estimates we propose new uniform controllability results for $1 \mathrm{D}$ semi-discrete parabolic equations and systems either for a distributed control or for a boundary control. They are proved by using the moments method that heavily depends on the discrete spectral properties established in this paper.

We first consider the semi-discretized controlled scalar parabolic equation with a finite time horizon $T>0$

$$
\left\{\begin{array}{l}
\left(y^{h}\right)^{\prime}(t)+\mathcal{A}^{h} y^{h}(t)=\mathcal{D}_{\omega}^{h} V_{\mathrm{d}}^{h}(t)+\mathcal{B}_{r}^{h} V_{\mathrm{b}}^{h}(t), \text { for } 0<t \leq T \\
y^{h}(0)=y^{0, h} \in \mathbb{R}^{N} .
\end{array}\right.
$$

Here, we consider two possible types of control

- $V_{\mathrm{d}}^{h}:(0, T) \rightarrow \mathbb{R}^{N}$ is a distributed control function that acts on the system through the control operator $\mathcal{D}_{\omega}^{h}$, which is the diagonal $N \times N$ matrix depending on the control domain $\omega$ and defined by

$$
\left(\mathcal{D}_{\omega}^{h}\right)_{i, i}:= \begin{cases}1 & \text { if } x_{i} \in \omega \\ 0 & \text { if } x_{i} \notin \omega\end{cases}
$$

- $V_{\mathrm{b}}^{h}:(0, T) \rightarrow \mathbb{R}$ is a boundary control function that acts on the system on the right boundary of the interval $\Omega$ (that is at $x=1$ ) through the control 
operator $\mathcal{B}_{r}^{h}$, which is the column vector defined by

$$
\mathcal{B}_{r}^{h}=\left(\begin{array}{c}
0 \\
\vdots \\
0 \\
\frac{\gamma_{N+1 / 2}}{h_{N} h_{N+1 / 2}}
\end{array}\right) .
$$

The particular form of this vector comes from the fact that, the matrix $\mathcal{A}^{h}$ was designed in (3) for homogeneous Dirichlet boundary condition and thus, we need to add a source term in the last component of the equation to account for a non-homogeneous Dirichlet boundary datum (which is precisely the control $V_{\mathrm{b}}^{h}$ we are looking for).

Of course, the same analysis can be carried out for a boundary control acting on the left boundary of $\Omega$, that is at $x=0$ but we will not give the details here.

System (6) is a discrete version of the following parabolic PDE control problem

$$
\left\{\begin{array}{l}
\partial_{t} y+\mathcal{A} y=1_{\omega} V_{\mathrm{d}}(t, x), \text { for } 0<t \leq T \\
y(t, 0)=0, \quad y(t, 1)=V_{\mathrm{b}}(t), \text { for } 0<t \leq T \\
y(0, .)=y^{0} \in L^{2}(\Omega)
\end{array}\right.
$$

The typical questions we are interested in concern null controllability and may be roughly formulated as follows (for a given set of data $\gamma, q, \omega, T, \ldots$ )

- Distributed control : Is there a constant $C>0$ such that for any $h>0$ small enough and any $y^{0, h} \in \mathbb{R}^{N}$ we can find a control $V_{\mathrm{d}}^{h} \in L^{2}\left(0, T, \mathbb{R}^{N}\right)$ such that the solution of (6) (with $V_{\mathrm{b}}^{h}=0$ ) satisfies $y^{h}(T)=0$ and moreover

$$
\left\|V_{\mathrm{d}}^{h}\right\|_{L^{2}\left(\Omega_{h}^{T}\right)} \leq C\left\|y^{0, h}\right\|_{L^{2}\left(\Omega_{h}\right)} .
$$

- Boundary control : Is there a constant $C>0$ such that for any, $h>0$ small enough, and any $y^{0, h} \in \mathbb{R}^{N}$, we can find a control $V_{\mathrm{b}}^{h} \in L^{2}(0, T)$ such that the solution of (6) (with $V_{\mathrm{d}}^{h}=0$ ) satisfies $y^{h}(T)=0$ and moreover

$$
\left\|V_{\mathrm{b}}^{h}\right\|_{L^{2}(0, T)} \leq C\left\|y^{0, h}\right\|_{L^{2}\left(\Omega_{h}\right)} .
$$

When $\gamma$ is constant, $q=0$ and for a uniform mesh, the boundary control case was positively solved in [17]. The authors use explicit formulas for $\lambda_{k}^{h}$ and $\phi_{k}^{h}$ in that case (see Remark 1.3) to tackle the problem of null controllability with the moments method. In the present work we propose to use the same kind of strategy but replacing the exact knowledge of the eigenelements of $\mathcal{A}^{h}$ by the estimates discussed above.

However, in the general case, it turns out that exact null-controllability at the discrete level formulated above is a too strong notion. Indeed, it may happen in some situations that the discrete problems are not uniformly controllable (we mention here the counter-example in 2D given in [21] for which (6) is not even approximately controllable for a given $h$ ). Finally, from a practical point of view, to obtain a bounded family of discrete controls that converges (at least weakly) to a null-control for the initial PDE problem, it is enough to weaken the requirement $y^{h}(T)=0, \forall h>0$ replacing it by the convergence $\left\|y^{h}(T)\right\|_{L^{2}\left(\Omega_{h}\right)} \rightarrow 0$ at a prescribed rate when $h \rightarrow 0$. Such a weaker requirement was already studied for instance in $[8,9,10,15]$ (yet with various naming conventions). A specific discussion on that topic was given in [7], from which we borrow the following definition. 
Definition 1.1. Let $\phi:(0,+\infty) \rightarrow(0,+\infty)$ be a function such that $\lim _{h \rightarrow 0} \phi(h)=$ 0 . We say that the family of Problems (6) is uniformly $\phi(h)$-null controlable with a distributed control (resp. a boundary control) if there exists a $C>0$, such that, for any $h$ small enough and any $y^{0, h} \in \mathbb{R}^{N}$, we can find a control $V_{d}^{h} \in L^{2}\left(0, T, \mathbb{R}^{N}\right)$ (resp. $V_{b}^{h} \in L^{2}(0, T)$ ) that satisfies

$$
\left\|V_{d}^{h}\right\|_{L^{2}\left(\Omega_{h}^{T}\right)} \leq C\left\|y^{0, h}\right\|_{L^{2}\left(\Omega_{h}\right)}, \quad\left(\text { resp. }\left\|V_{b}^{h}\right\|_{L^{2}(0, T)} \leq C\left\|y^{0, h}\right\|_{L^{2}\left(\Omega_{h}\right)}\right),
$$

and such that the solution $y^{h}$ of (6) with $V_{b}^{h}=0$ (resp. $V_{d}^{h}=0$ ) satisfies

$$
\left\|y^{h}(T)\right\|_{L^{2}\left(\Omega_{h}\right)}^{2} \leq C \phi(h)\left\|y^{0, h}\right\|_{L^{2}\left(\Omega_{h}\right)}^{2} .
$$

It is shown in $[8,9,10]$ that, for a finite difference approximation on smooth grids with smooth coefficients, the uniform $\phi(h)$-null controllability property holds for a distributed control as soon as $\phi$ does not tend to zero faster that some exponential $h \mapsto e^{-C / h^{\alpha}}$ (see also [15] where the case of Galerkin approximation is discussed). However, in those references, the methods which are used (based on discrete Carleman estimates) do not allow to tackle the case of coupled parabolic systems with a boundary control.

With the techniques developped in the present paper (however restricted to the 1D case), we are able to obtain similar results for the scalar equation (6) both for distributed and boundary control problems, as well as for the coupled systems presented below, which is, up to our knowledge, the first result in that framework.

A typical semi-discrete parabolic system with two components we shall deal with is the following

$\left(S_{2}^{h}\right)\left\{\begin{array}{l}\left(Y^{h}\right)^{\prime}(t)+\left(\begin{array}{cc}\mathcal{A}^{h} & 0^{h} \\ \mathrm{I}^{h} & \mathcal{A}^{h}\end{array}\right) Y^{h}(t)=\left(\begin{array}{c}\mathrm{I}^{h} \\ 0^{h}\end{array}\right)\left(\mathcal{D}_{\omega}^{h} V_{\mathrm{d}}^{h}(t)+\mathcal{B}_{r}^{h} V_{\mathrm{b}}^{h}(t)\right), \text { for } 0<t \leq T, \\ Y^{h}(0)=Y^{0, h} \in\left(\mathbb{R}^{N}\right)^{2} .\end{array}\right.$

In this system, $\mathrm{I}^{h}$ (resp. $0^{h}$ ) is the identity matrix (resp. the zero matrix) of size $N \times N$. The unknown $Y^{h}(t) \in\left(\mathbb{R}^{N}\right)^{2}$ has now two components. The first one satisfies the same scalar equation as before and is controlled either by a distributed control $V_{\mathrm{d}}^{h}$ or by a boundary control $V_{\mathrm{b}}^{h}$, whereas the second component satisfies a parabolic equation without explicit appearance of a control; actually this component of the solution is indirectly controlled by the first component itself by means of the coupling term which is here set to 1 and which appears in the matrix $\left(\begin{array}{cc}\mathcal{A}^{h} & 0^{h} \\ \mathrm{I}^{h} & \mathcal{A}^{h}\end{array}\right)$. The controllability questions we are interested in are now exactly the same: is it possible to find a bounded family of controls $\left(V_{\mathrm{d}}^{h}\right)_{h}$ (resp. $\left.\left(V_{\mathrm{b}}^{h}\right)_{h}\right)$ such that the two components of the corresponding solution of $\left(S_{2}^{h}\right)$ are small at time $T$, that is

$$
\left\|Y^{h}(T)\right\|_{L^{2}\left(\Omega_{h}\right)}^{2} \leq C \phi(h)\left\|Y^{0, h}\right\|_{L^{2}\left(\Omega_{h}\right)}^{2},
$$

where the notation for the norms $\|\cdot\|_{L^{2}\left(\Omega_{h}\right)}$ are adapted to the fact that $Y^{h}$ has now two components. The corresponding precise theorems are stated in Section 5.3.

To conclude this introduction, we mention that we do not address here the question of the actual computation of the controls. Even though we shall use the moments method in the analysis, it has to be precised that this is not at all a constructive method that can be efficiently implemented. Instead, once a theoretical uniform $\phi(h)$ null controllability result is obtained, we can implement the penalised HUM method that provides, in a more natural and generic way, discrete controls that fulfills the properties of definition 1.1. This approach is discussed for instance in [7, 14]. 
2. Spectral properties of elliptic operators. The continuous case. In this section we present a proof of Theorem 1.1 even though this result is not new by itself (see for instance [19]). Our goal is just to introduce a quite simple proof that we will manage to adapt to the discrete setting in the following sections. In all this section, we assume that hypothesis $\left(\mathrm{H}_{0}\right)$ holds.

Lemma 2.1. Let $\omega$ be a non-empty open subset of $\Omega$. There exists $C_{1}(q, \gamma)>0$ and $C_{2}(q, \gamma, \omega)>0$ such that we have, for any $k \geq 1$,

$$
\frac{1}{\lambda_{k}}\left|\partial_{b} \phi_{k}\right|^{2} \geq C_{1} \mathcal{R}_{k}, \quad \forall b \in\{l, r\}
$$

and

where we have defined

$$
\left\|\phi_{k}\right\|_{L^{2}(\omega)}^{2} \geq C_{2} \mathcal{R}_{k}
$$

$$
\mathcal{R}_{k}:=\inf _{x, y \in \Omega} \frac{\left|\phi_{k}(x)\right|^{2}+\frac{\gamma(x)}{\lambda_{k}}\left|\phi_{k}^{\prime}(x)\right|^{2}}{\left|\phi_{k}(y)\right|^{2}+\frac{\gamma(y)}{\lambda_{k}}\left|\phi_{k}^{\prime}(y)\right|^{2}} .
$$

This lemma says, in particular, that to obtain (possibly uniform in $k$ ) lower bounds on $\left|\partial_{b} \phi_{k}\right| / \sqrt{\lambda_{k}}$ and $\left\|\phi_{k}\right\|_{L^{2}(\omega)}$, it is enough to obtain lower bounds on $\mathcal{R}_{k}$. Its proof is postponed at the end of the section.

Our strategy is based on interpreting the equation satisfied by eigenfunctions as a particular first order ordinary differential system in such a way that the principal part (with respect to the large values of $\lambda_{k}$ ) of the evolution matrix is skew-symmetric. It will let us obtain suitable estimates on the quantity $\mathcal{R}_{k}$ defined in the previous lemma. In this direction, we begin with the following lemma whose proof is a straightforward computation and is left to the reader.

Lemma 2.2. Let $f: \Omega \rightarrow \mathbb{R}$ be a continuous function and $\lambda>0$. Let $u \in \mathcal{C}^{2}(\Omega)$ be a real valued function satisfying the second-order differential equation (without any assumption on boundary conditions)

$$
\mathcal{A} u(x)=\lambda u(x)+f(x), \quad \forall x \in \Omega,
$$

then the following equation holds

$$
U^{\prime}(x)=M(x) U(x)+Q(x) U(x)+F(x),
$$

where we have defined the vectors

$$
U(x):=\left(\begin{array}{c}
u(x) \\
\sqrt{\frac{\gamma(x)}{\lambda}} u^{\prime}(x)
\end{array}\right) \text { and } F(x):=\left(\begin{array}{c}
0 \\
-\frac{f(x)}{\sqrt{\gamma(x) \lambda}}
\end{array}\right) .
$$

and the matrices

$$
M(x):=\left(\begin{array}{cc}
0 & \sqrt{\frac{\lambda}{\gamma(x)}} \\
-\sqrt{\frac{\lambda}{\gamma(x)}} & 0
\end{array}\right) \text { and } Q(x):=\left(\begin{array}{cc}
0 & 0 \\
\frac{q(x)}{\sqrt{\lambda \gamma(x)}} & \sqrt{\gamma(x)}\left(\frac{1}{\sqrt{\gamma}}\right)^{\prime}(x)
\end{array}\right) .
$$

The key-point of this formulation is that the large terms in $\sqrt{\lambda}$ only appear in the skew-symmetric matrix $M(x)$, while the matrix $Q(x)$ only contains bounded terms with respect to $\lambda$.

As a consequence of this particular structure, we can obtain the following estimates. 
LEMMA 2.3. With the same notations as in Lemma 2.2, and assuming that $\lambda \geq 1$, there exists $C:=C(\gamma, q)$, independent of $\lambda$, such that for any $x, y \in \Omega$, we have

$$
\|U(y)\| \leq C\left(\|U(x)\|+\left|\int_{x}^{y}\|F(s)\| \mathrm{d} s\right|\right) .
$$

Proof. Let $x, y \in \Omega$. It is fundamental to notice that the matrices $(M(s))_{s}$ pairwise commute, so that the resolvant operator associated with $x \mapsto M(x)$ simply reads

$$
S(y, x):=\exp \left(\int_{x}^{y} M(s) \mathrm{d} s\right)
$$

We can then use Duhamel's formula to deduce from equation (10) the following expression

$$
U(y)=S(y, x) U(x)+\int_{x}^{y} S(y, s)(Q(s) U(s)+F(s)) \mathrm{d} s .
$$

We use now the fact that the matrix $M(s)$ is skew-symmetric for any $s$, and so is $\int_{x}^{y} M(s) \mathrm{d} s$. It follows that the resolvant $S(y, s)$ satisfies $\|S(y, s)\|=1$ for any $y, s$. We get

$$
\|U(y)\| \leq\|U(x)\|+\left|\int_{x}^{y}\|F(s)\| \mathrm{d} s\right|+\left|\int_{x}^{y}\|Q(s)\|\|U(s)\| \mathrm{d} s\right| .
$$

Gronwall's lemma finally yields

$$
\|U(y)\| \leq\left(\|U(x)\|+\left|\int_{x}^{y}\|F(s)\| \mathrm{d} s\right|\right) \exp \left(\left|\int_{x}^{y}\|Q(s)\| \mathrm{d} s\right|\right),
$$

which gives the result since $Q(s)$ is bounded uniformly in $s$ and $\lambda$, by using assumption $\left(\mathrm{H}_{0}\right)$.

We can now prove Theorem 1.1. Note that it is enough to prove the claims for $k$ large enough and in particular, by (2), we can assume without any loss of generality that $\lambda_{k} \geq 1$.

- We begin with the proof of points 2. and 3. of the theorem, that is the properties which concern the eigenfunctions $\left(\phi_{k}\right)$. By definition, $\phi_{k}$ is a solution of the equation

$$
\mathcal{A} \phi_{k}=\lambda_{k} \phi_{k}
$$

which is exactly (9) with $u=\phi_{k}, \lambda=\lambda_{k}, f=0$. From Lemma 2.3 we deduce that there exists $C:=C(\gamma, q)$, independent of $k$, such that for any $x, y \in \bar{\Omega}$,

$$
\left|\phi_{k}(y)\right|^{2}+\frac{\gamma(y)}{\lambda_{k}}\left|\phi_{k}^{\prime}(y)\right|^{2} \geq C\left(\left|\phi_{k}(x)\right|^{2}+\frac{\gamma(x)}{\lambda_{k}}\left|\phi_{k}^{\prime}(x)\right|^{2}\right),
$$

which exactly proves that the quantity $\mathcal{R}_{k}$ defined in (8) is uniformly bounded from below. The claim thus immediately follows from Lemma 2.1.

- We shall now prove the first point in Theorem 1.1. For any index $k \geq 1$ with $\lambda_{k} \geq 1$, we define

$$
u(x):=\phi_{k}^{\prime}(1) \phi_{k+1}(x)-\phi_{k+1}^{\prime}(1) \phi_{k}(x),
$$

in such a way that $u(1)=u^{\prime}(1)=0$ and

$$
\mathcal{A} u=\lambda_{k+1} u+f,
$$


SPECTRAL ANALYSIS OF DISCRETE ELLIPTIC OPERATORS AND APPLICATIONS 13

with

$$
f(x):=\phi_{k+1}^{\prime}(1) \phi_{k}(x)\left(\lambda_{k+1}-\lambda_{k}\right) .
$$

Using the notations introduced in Lemma 2.2 , we observe that by construction we have $U(1)=0$ so that the estimate (11) specialized in $x=1$ leads to

$$
\|U(y)\| \leq C \int_{y}^{1}\|F(s)\| \mathrm{d} s \leq C \int_{0}^{1}\|F(s)\| \mathrm{d} s, \quad \forall y \in \Omega .
$$

Using the expression for $F$ and $f$, we find that

$$
\|U(y)\| \leq \frac{C}{\sqrt{\gamma_{\min }}}\left(\frac{\lambda_{k+1}-\lambda_{k}}{\sqrt{\lambda_{k+1}}}\left|\phi_{k+1}^{\prime}(1)\right|\right) \int_{0}^{1}\left|\phi_{k}(s)\right| \mathrm{d} s, \forall y \in \Omega .
$$

Thanks to the normalisation condition $\left\|\phi_{k}\right\|_{L^{2}(\Omega)}=1$ and the expressions of $U$ and $u$, we obtain for any $y \in \Omega$,

$$
\left|\phi_{k}^{\prime}(1) \phi_{k+1}(y)-\phi_{k+1}^{\prime}(1) \phi_{k}(y)\right|^{2} \leq \frac{C}{\gamma_{\min }}\left(\frac{\lambda_{k+1}-\lambda_{k}}{\sqrt{\lambda_{k+1}}}\left|\phi_{k+1}^{\prime}(1)\right|\right)^{2} .
$$

We integrate this inequality with respect to $y \in(0,1)$ and we use the $L^{2}(\Omega)$ orthonormality of $\phi_{k+1}$ and $\phi_{k}$ to finally get

$$
\left|\phi_{k+1}^{\prime}(1)\right|^{2} \leq\left(\phi_{k+1}^{\prime}(1)\right)^{2}+\left(\phi_{k}^{\prime}(1)\right)^{2} \leq \frac{C}{\gamma_{\min }}\left(\frac{\lambda_{k+1}-\lambda_{k}}{\sqrt{\lambda_{k+1}}}\left|\phi_{k+1}^{\prime}(1)\right|\right)^{2},
$$

and since $\phi_{k+1}^{\prime}(1) \neq 0$, we conclude that

$$
\lambda_{k+1}-\lambda_{k} \geq \bar{C} \sqrt{\lambda_{k+1}},
$$

for some $\bar{C}(\gamma, q)>0$ independent of $k$. The claim follows by the lower bound in (2).

REMARK 2.1. Integrating (13) with respect to $y$ over $\Omega$ and using the min-max principle, we can easily obtain optimal $L^{\infty}$ bounds on the eigenfunctions

$$
\left\|\phi_{k}\right\|_{L^{\infty}(\Omega)} \leq C, \quad\left\|\phi_{k}^{\prime}\right\|_{L^{\infty}(\Omega)} \leq C \sqrt{\lambda_{k}}, \quad \forall k \geq 1 .
$$

REMARK 2.2. Observe that the only point where the regularity of $\gamma$ is used in this proof is in the uniform estimate of $\|Q(s)\|$. However, the only thing we need is that $\int_{\Omega}\|Q(s)\| d s<+\infty$, which proves that Theorem 1.1 still holds when $\gamma$ is piecewise $\mathcal{C}^{1}$.

It remains to give the proof of the lemma. Using Caccioppoli-like inequality or nodal sets of eigenfunctions of Sturm-Liouville operators, one can give a more direct proof of this lemma. The advantage of the proof given here is that it can be more easily extended to the discrete setting (see Section 3.4).

Proof (of Lemma 2.1). From (8) we can write

$$
\mathcal{R}_{k}\left(\left|\phi_{k}(y)\right|^{2}+\frac{\gamma(y)}{\lambda_{k}}\left|\phi_{k}^{\prime}(y)\right|^{2}\right) \leq\left|\phi_{k}(x)\right|^{2}+\frac{\gamma(x)}{\lambda_{k}}\left|\phi_{k}^{\prime}(x)\right|^{2}, \forall x, y \in \Omega .
$$


We recall that $\phi_{k}$ satisfies the Dirichlet boundary condition $\phi_{k}(0)=\phi_{k}(1)=0$, so that by choosing $x=0$ and $x=1$ in the previous inequality, we obtain in particular

$$
\frac{\gamma(0)}{\lambda_{k}}\left|\partial_{l} \phi_{k}\right|^{2} \geq \mathcal{R}_{k}\left|\phi_{k}(y)\right|^{2}, \forall y \in \Omega,
$$

and

$$
\frac{\gamma(1)}{\lambda_{k}}\left|\partial_{r} \phi_{k}\right|^{2} \geq \mathcal{R}_{k}\left|\phi_{k}(y)\right|^{2}, \forall y \in \Omega \text {. }
$$

Since the left-hand side does not depend on $y$, and thanks to the normalisation condition $\left\|\phi_{k}\right\|_{L^{2}(\Omega)}=1$, we can integrate those inequalities with respect to $y$ to obtain

$$
\frac{\gamma(0)}{\lambda_{k}}\left|\partial_{l} \phi_{k}\right|^{2} \geq \mathcal{R}_{k}, \quad \text { and } \quad \frac{\gamma(1)}{\lambda_{k}}\left|\partial_{r} \phi_{k}\right|^{2} \geq \mathcal{R}_{k} .
$$

Consider now any non empty open subset $\omega \subset \Omega$. Without any loss of generality we can assume that $\omega$ is a non-empty interval. Split $\omega$ in three consecutive disjoint intervals of identical measure : $\omega_{1}, \omega_{2}$ and $\omega_{3}$.

- Suppose that there exists $a_{k} \in \omega_{1}$ and $b_{k} \in \omega_{3}$ such that

$$
\phi_{k}\left(a_{k}\right) \phi_{k}^{\prime}\left(a_{k}\right) \geq 0 \text { and } \phi_{k}\left(b_{k}\right) \phi_{k}^{\prime}\left(b_{k}\right) \leq 0 .
$$

We multiply the equation $\mathcal{A} \phi_{k}=\lambda_{k} \phi_{k}$ by $\phi_{k}$ and integrate by parts on $\left(a_{k}, b_{k}\right)$. We get

$$
-\underbrace{\left[\gamma(x) \phi_{k}^{\prime}(x) \phi_{k}(x)\right]_{a_{k}}^{b_{k}}}_{\leq 0}+\int_{a_{k}}^{b_{k}}\left(\gamma(x)\left(\phi_{k}^{\prime}(x)\right)^{2}+q(x) \phi_{k}^{2}(x)\right) d x=\lambda_{k} \int_{a_{k}}^{b_{k}} \phi_{k}^{2}(x) d x
$$

thus,

$$
\int_{a_{k}}^{b_{k}} \gamma(x)\left(\phi_{k}^{\prime}(x)\right)^{2} d x \leq\left(\lambda_{k}+\|q\|_{\infty}\right) \int_{a_{k}}^{b_{k}} \phi_{k}^{2}(x) d x
$$

We come back to (14) that we integrate on the whole domain $\Omega$ with respect to the variable $y$ and on the interval $\left(a_{k}, b_{k}\right)$ with respect to the variable $x$. With the normalisation condition on $\phi_{k}$ and (16) we obtain:

$$
\mathcal{R}_{k}\left(b_{k}-a_{k}\right) \leq \int_{a_{k}}^{b_{k}} \phi_{k}^{2}(x)+\frac{\gamma(x)}{\lambda_{k}}\left(\phi_{k}^{\prime}(x)\right)^{2} d x \leq\left(2+\frac{\|q\|_{\infty}}{\lambda_{1}}\right) \int_{a_{k}}^{b_{k}} \phi_{k}^{2}(x) d x .
$$

Using that $a_{k} \in \omega_{1}$ and $b_{k} \in \omega_{3}$ we get $b_{k}-a_{k} \geq\left|\omega_{2}\right|=\frac{|\omega|}{3}$ and we finally obtain the claimed lower bound

$$
\mathcal{R}_{k} \frac{|\omega|}{3} \leq \mathcal{R}_{k}\left(b_{k}-a_{k}\right) \leq C \int_{\omega} \phi_{k}^{2}(x) d x .
$$

- Suppose now that either

$$
\forall x \in \omega_{1}, \phi_{k}(x) \phi_{k}^{\prime}(x)<0
$$

Or

$$
\forall x \in \omega_{3}, \phi_{k}(x) \phi_{k}^{\prime}(x)>0,
$$

hold. We only consider the second case (18), since the case (17) is similar. 
REMARK 2.3. Notice that from condition (18), we can deduce that either $\phi_{k}$ is positive and increasing on $\omega_{3}$ or $\phi_{k}$ is negative and decreasing on $\omega_{3}$. Note also that this condition implies that $: x \rightarrow|\phi|(x)$ is strictly increasing on $\omega_{3}$.

We split $\omega_{3}$ in two consecutive subintervals $\omega_{3,1}$ and $\omega_{3,2}$ of identical measure.

First case : Suppose that there exists $x_{0} \in \omega_{3,1}$ such that $\frac{\gamma\left(x_{0}\right)}{\lambda_{k}}\left|\phi_{k}^{\prime}\left(x_{0}\right)\right|^{2} \leq$ $\frac{\mathcal{R}_{k}}{2}$. This assumption and inequality (14) give

$$
\left|\phi_{k}\left(x_{0}\right)\right|^{2}+\frac{\mathcal{R}_{k}}{2} \geq\left|\phi_{k}\left(x_{0}\right)\right|^{2}+\frac{\gamma\left(x_{0}\right)}{\lambda_{k}}\left|\phi_{k}^{\prime}\left(x_{0}\right)\right|^{2} \geq \mathcal{R}_{k}\left|\phi_{k}(y)\right|^{2}, \forall y \in \Omega,
$$

and integrating on $\Omega$ with respect to the variable $y$, we get:

$$
\left|\phi_{k}\left(x_{0}\right)\right|^{2} \geq \frac{\mathcal{R}_{k}}{2}
$$

According to Remark 2.3, $|\phi|^{2}$ is increasing on $\omega_{3}$. Thus, integrating on $\omega_{3,2}$, we obtain

$$
\int_{\omega_{3,2}}\left|\phi_{k}(x)\right|^{2} d x \geq C \mathcal{R}_{k}|\omega|
$$

Second case: Suppose now that $\forall x_{0} \in \omega_{3,1}, \frac{\gamma\left(x_{0}\right)}{\lambda_{k}}\left|\phi_{k}^{\prime}\left(x_{0}\right)\right|^{2}>\frac{\mathcal{R}_{k}}{2}$. Let us integrate the square root of this inequality on $\omega_{3,1}$

$$
\int_{\omega_{3,1}}\left|\phi_{k}^{\prime}\left(x_{0}\right)\right| d x_{0}>C \sqrt{\lambda_{k} \mathcal{R}_{k}}\left|\omega_{3,1}\right|
$$

According to Remark 2.3, $\phi_{k}$ is monotonic on $\omega_{3}$ so $\int_{\omega_{3,1}}\left|\phi_{k}^{\prime}\left(x_{0}\right)\right| d x_{0}=$ $\left|\int_{\omega_{3,1}} \phi_{k}^{\prime}\left(x_{0}\right) d x_{0}\right|$. Denote by $a_{3,1}<b_{3,1}$ the end points of $\omega_{3,1}$. We have

$$
\left|\phi_{k}\left(b_{3,1}\right)-\phi_{k}\left(a_{3,1}\right)\right|>C \sqrt{\lambda_{k} \mathcal{R}_{k}}\left|\omega_{3,1}\right|,
$$

and thus, since $\left|\phi_{k}\right|$ is increasing,

$$
2\left|\phi_{k}\left(b_{3,1}\right)\right|>C \sqrt{\lambda_{k} \mathcal{R}_{k}}\left|\omega_{3,1}\right|
$$

Hence,

$$
\int_{\omega}\left|\phi_{k}\right|^{2}(x) d x \geq \int_{\omega_{3,2}}\left|\phi_{k}\right|^{2}(x) d x \geq\left|\phi_{k}\left(b_{3,1}\right)\right|^{2}\left|\omega_{3,2}\right| \geq C \lambda_{k} \mathcal{R}_{k}|\omega|^{3},
$$

which concludes the proof.

\section{Discrete spectral properties.}

3.1. General strategy. As previously stated, our goal here is to prove qualitative properties for the eigenelements of the discrete elliptic operator $\mathcal{A}^{h}$. We shall adapt Theorem 1.1 to the discrete setting by mimicking the proof given in Section 2 . We start by the following preliminary result. Its proof is postponed to Section 3.4. We recall that we have assumed that $q \geq 0$. 
Lemma 3.1. Assume that $\left(\mathrm{H}_{0}\right)$ holds. Let $\omega$ be a non-empty open subset of $\Omega$ and $\beta>0$. There exist $h_{1}(q, \gamma, \beta)>0, h_{2}(q, \gamma, \omega, \beta)>0$ and $C_{1}(q, \gamma, \beta)>0$, $C_{2}(q, \gamma, \omega, \beta)>0$ such that for any mesh of $\Omega$ such that $\Theta_{h} \leq \beta$, we have

$$
\frac{1}{\lambda_{k}^{h}}\left|\partial_{b} \phi_{k}^{h}\right|^{2} \geq C_{1} \mathcal{R}_{k}^{h}, \quad \forall b \in\{l, r\}, \forall k \in \llbracket 1, N \rrbracket,
$$

if $h \leq h_{1}$ and

$$
\left\|\phi_{k}^{h}\right\|_{L^{2}\left(\omega_{h}\right)}^{2} \geq C_{2} \mathcal{R}_{k}^{h}, \forall k \in \llbracket 1, N \rrbracket,
$$

if $h \leq h_{2}$, with $\mathcal{R}_{k}^{h}$ given by

$$
\mathcal{R}_{k}^{h}:=\min _{i, j \in \llbracket 1, N+1 \rrbracket} \frac{\left|\phi_{i, k}^{h}\right|^{2}+\frac{\gamma_{i-1 / 2}}{\lambda_{k}^{h}}\left|\frac{\phi_{i, k}^{h}-\phi_{i-1, k}^{h}}{h_{i-1 / 2}}\right|^{2}}{\left|\phi_{j, k}^{h}\right|^{2}+\frac{\gamma_{j-1 / 2}}{\lambda_{k}^{h}}\left|\frac{\phi_{j, k}^{h}-\phi_{j-1, k}^{h}}{h_{j-1 / 2}}\right|^{2}} .
$$

This lemma shows that, to obtain (possibly uniform in $k$ and $h$ ) lower bounds on $\left|\partial_{b} \phi_{k}^{h}\right| / \sqrt{\lambda_{k}^{h}}$ and $\left\|\phi_{k}^{h}\right\|_{L^{2}\left(\omega_{h}\right)}$ it is enough to obtain lower bounds on $\mathcal{R}_{k}^{h}$.

To this end, we propose the following transformation of the discrete second order equation into a suitable first order system.

LEMMA 3.2. We consider a given mesh of $\Omega, f^{h}$ a vector of $\mathbb{R}^{N}, \lambda>0$ and we assume that $u^{h} \in \mathbb{R}^{N}$ satisfies the discrete equation

$$
\mathcal{A}^{h} u^{h}=\lambda u^{h}+f^{h}
$$

Then, the following relation holds for any $j \in \llbracket 1, N \rrbracket$

$$
U_{j+1}^{h}=\left(\mathrm{I}+h_{j} M_{j}^{h}\right) U_{j}^{h}+h_{j}\left(Q_{j}^{h} U_{j}^{h}+F_{j}^{h}\right),
$$

where we have defined the families of vectors

$$
U_{j}^{h}:=\left(\begin{array}{c}
u_{j}^{h} \\
\sqrt{\frac{\gamma_{j-1 / 2}}{\lambda}} \frac{u_{j}^{h}-u_{j-1}^{h}}{h_{j-1 / 2}}
\end{array}\right) \text { and } F_{j}^{h}:=\left(\begin{array}{c}
-h_{j+1 / 2} \frac{f_{j}^{h}}{\gamma_{j+1 / 2}} \\
-\frac{f_{j}^{h}}{\sqrt{\gamma_{j+1 / 2} \lambda}}
\end{array}\right)
$$

as well as the families of matrices

$$
M_{j}^{h}:=\left(\begin{array}{cc}
-h_{j-1 / 2 \frac{\lambda}{\gamma_{j+1 / 2}}} & \frac{h_{j+1 / 2}}{h_{j}} \sqrt{\frac{\lambda}{\gamma_{j+1 / 2}}} \\
-\sqrt{\frac{\lambda}{\gamma_{j+1 / 2}}} & 0
\end{array}\right),
$$

and

$$
Q_{j}^{h}:=\left(\begin{array}{cc}
h_{j+1 / 2} \frac{q_{j}}{\gamma_{j+1 / 2}} & h_{j+1 / 2} \sqrt{\frac{\lambda \gamma_{j-1 / 2}}{\gamma_{j+1 / 2}} \frac{1}{\sqrt{\gamma_{j+1 / 2}}}-\frac{1}{\sqrt{\gamma_{j-1 / 2}}}} \frac{h_{j}}{\sqrt{\gamma_{j+1 / 2}}-\frac{1}{\sqrt{\gamma_{j-1 / 2}}}} \\
\frac{q_{j}}{\sqrt{\lambda \gamma_{j+1 / 2}}} & \sqrt{\gamma_{j-1 / 2}} \frac{\sqrt{\gamma_{j}}}{}
\end{array}\right) .
$$

REMARK 3.1. Note that the matrix $\left(\mathrm{I}+h_{j} M_{j}^{h}\right)$ is a one step propagator which is the discrete analogue of $\exp \left(\int_{x_{j}}^{x_{j+1}} M(s) \mathrm{d} s\right)$.

We shall make use of the following estimates. 
SPECTRAL ANALYSIS OF DISCRETE ELLIPTIC OPERATORS AND APPLICATIONS 17

Lemma 3.3. For any mesh of size $N$ and any $\gamma$, q satisfying $\left(\mathrm{H}_{0}\right)$, we have the inequalities

$$
\frac{\gamma_{\min }}{\left(\Theta_{h}\right)^{2}} \lambda_{k}^{h_{*}}-\|q\|_{L^{\infty}} \leq \lambda_{k}^{h} \leq\left(\Theta_{h}\right)^{2}\|\gamma\|_{L^{\infty}} \lambda_{k}^{h_{*}}+\|q\|_{L^{\infty}}, \quad \forall k \in \llbracket 1, N \rrbracket,
$$

where $\lambda_{k}^{h_{*}}$ was defined in Remark 1.3. In particular, for any $\beta>0$, there exists $C_{1}, C_{2}, C_{3}>0$ depending only on $\gamma, q$ and $\beta$ such that for any mesh satisfying $\Theta_{h} \leq \beta$ we have the inequalities

$$
\begin{aligned}
C_{1} k^{2}-C_{2} \leq \lambda_{k}^{h} \leq & C_{3} k^{2}+C_{2}, \quad \forall k \in \llbracket 1, N \rrbracket, \\
& h^{2} \lambda_{k}^{h} \leq C_{2}, \quad \forall k \in \llbracket 1, N \rrbracket .
\end{aligned}
$$

The proof relies on Remark 1.3 and the min-max principle. It is a straightforward computation that we leave to the reader. Those estimates can be seen as discrete versions of (2).

Proof (of lemma 3.2). The proof of (21) is a tedious but simple computation. The second component of $U_{j+1}^{h}-U_{j}^{h}$ satisfies

$$
\begin{aligned}
\sqrt{\frac{\gamma_{j+1 / 2}}{\lambda} \frac{u_{j+1}^{h}-u_{j}^{h}}{h_{j+1 / 2}}-} & \sqrt{\frac{\gamma_{j-1 / 2}}{\lambda}} \frac{u_{j}^{h}-u_{j-1}^{h}}{h_{j-1 / 2}} \\
= & \frac{1}{\sqrt{\lambda \gamma_{j+1 / 2}}}\left(\gamma_{j+1 / 2} \frac{u_{j+1}^{h}-u_{j}^{h}}{h_{j+1 / 2}}-\gamma_{j-1 / 2} \frac{u_{j}^{h}-u_{j-1}^{h}}{h_{j-1 / 2}}\right) \\
& \quad+\frac{u_{j}^{h}-u_{j-1}^{h}}{h_{j-1 / 2}} \sqrt{\frac{\gamma_{j-1 / 2}}{\lambda}} \sqrt{\gamma_{j-1 / 2}}\left(\frac{1}{\sqrt{\gamma_{j+1 / 2}}}-\frac{1}{\sqrt{\gamma_{j-1 / 2}}}\right) \\
=- & h_{j}\left(\frac{\lambda-q_{j}}{\sqrt{\lambda \gamma_{j+1 / 2}}}\right) u_{j}^{h}-h_{j} \frac{f_{j}^{h}}{\sqrt{\lambda \gamma_{j+1 / 2}}} \\
& +\frac{u_{j}^{h}-u_{j-1}^{h}}{h_{j-1 / 2}} \sqrt{\frac{\gamma_{j-1 / 2}}{\lambda}} h_{j} \sqrt{\gamma_{j-1 / 2}}\left(\frac{\frac{1}{\sqrt{\gamma_{j+1 / 2}}}-\frac{1}{\sqrt{\gamma_{j-1 / 2}}}}{h_{j}}\right),
\end{aligned}
$$

where we used the original equation (20) in the last equality, whereas for the first component, we simply write

$$
u_{j+1}^{h}-u_{j}^{h}=\left(h_{j+1 / 2} \sqrt{\frac{\lambda}{\gamma_{j+1 / 2}}}\right) \sqrt{\frac{\gamma_{j+1 / 2}}{\lambda}} \frac{u_{j+1}^{h}-u_{j}^{h}}{h_{j+1 / 2}} .
$$

It follows, in vectorial form, the following equality

$$
\begin{aligned}
& U_{j+1}^{h}-U_{j}^{h}=\left(\begin{array}{cc}
0 & h_{j+1 / 2} \sqrt{\frac{\lambda}{\gamma_{j+1 / 2}}} \\
0 & 0
\end{array}\right) U_{j+1}^{h}+\left(\begin{array}{cc}
0 & 0 \\
-h_{j} \sqrt{\frac{\lambda}{\gamma_{j+1 / 2}}} & 0
\end{array}\right) U_{j}^{h}
\end{aligned}
$$

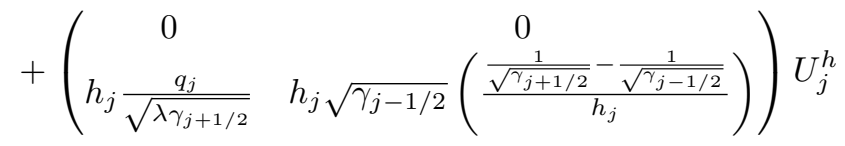

$$
\begin{aligned}
& +\left(\begin{array}{c}
0 \\
-h_{j} \frac{f_{j}^{h}}{\sqrt{\gamma_{j+1 / 2} \lambda}}
\end{array}\right) \text {. }
\end{aligned}
$$


This is not yet the required form since $U_{j+1}^{h}$ appears in the right-hand side. Thus, we collect the terms in $U_{j+1}^{h}$ in the left-hand side as follows

$$
\begin{aligned}
& \left(\begin{array}{cc}
1 & -h_{j+1 / 2} \sqrt{\frac{\lambda}{\gamma_{j+1 / 2}}} \\
0 & 1
\end{array}\right) U_{j+1}^{h}=\left(\begin{array}{cc}
1 & 0 \\
-h_{j} \sqrt{\frac{\lambda}{\gamma_{j+1 / 2}}} & 1
\end{array}\right) U_{j}^{h}
\end{aligned}
$$

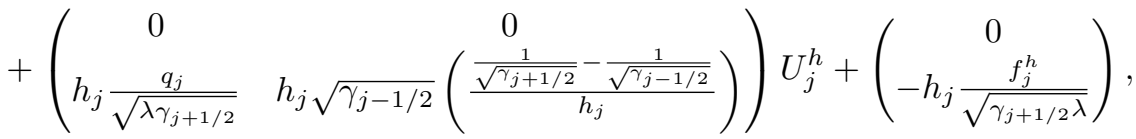

and we get the claim by multiplying the previous equality by

$$
\left(\begin{array}{cc}
1 & h_{j+1 / 2} \sqrt{\frac{\lambda}{\gamma_{j+1 / 2}}} \\
0 & 1
\end{array}\right)
$$

which is nothing but the inverse of the $2 \times 2$ matrix appearing in the left-hand side term.

For any $j, k \in \llbracket 1, N \rrbracket$, we denote by $Q_{j, k}^{h}, M_{j, k}^{h}$ the matrices as defined in the previous lemma for the particular value $\lambda=\lambda_{k}^{h}$. Using the uniform bounds on the discrete eigenvalues obtained in Lemma 3.3, one can easily deduce that there exists a $C\left(q, \gamma, \Theta_{h}\right)>0$ but independent of $h$ such that

$$
\left\|Q_{j, k}^{h}\right\| \leq C, \text { and }\left\|M_{j, k}^{h}\right\| \leq C \sqrt{\lambda_{k}^{h}}, \quad \forall h>0, \forall j, k \in \llbracket 1, N \rrbracket,
$$

and moreover, if $\gamma$ is assumed to be constant then we have the additional estimate

$$
\left\|Q_{j, k}^{h}\right\| \leq C\left(h_{j+1 / 2}+\frac{1}{\sqrt{\lambda_{k}^{h}}}\right), \quad \forall h>0, \forall j, k \in \llbracket 1, N \rrbracket .
$$

3.2. Estimates in the general case (S1). We are now in position to prove our first results in the more general case we shall consider, that is the setting (S1).

THEOREM 3.1. Let $\gamma$ and $q$ satisfying $\left(\mathrm{H}_{0}\right), \omega$ a non empty open subset of $\Omega$ and $\beta>0$. There exists $h_{1}(q, \gamma, \beta)>0, h_{2}(q, \gamma, \omega, \beta)>0$ and $C(q, \gamma, \beta)>0$, $C_{1}(q, \gamma, \beta)>0, C_{2}(q, \gamma, \omega, \beta)>0$ such that the following lower bounds hold for any mesh of $\Omega$ such that $\Theta_{h} \leq \beta$

$$
\frac{1}{\sqrt{\lambda_{k}^{h}}}\left|\partial_{b} \phi_{k}^{h}\right| \geq C_{1} e^{-C \sqrt{\lambda_{k}^{h}}}, \forall k \in \llbracket 1, N \rrbracket, \forall b \in\{l, r\},
$$

as soon as $h \leq h_{1}$ and

$$
\left\|\phi_{k}^{h}\right\|_{L^{2}\left(\omega_{h}\right)}^{2} \geq C_{2} e^{-C \sqrt{\lambda_{k}^{h}}}, \forall k \in \llbracket 1, N \rrbracket,
$$

as soon as $h \leq h_{2}$.

Moreover, if $\gamma$ and $q$ satisfy hypothesis $\left(\mathrm{H}_{2}\right)$, there exists $C_{3}(q, \gamma, \beta)>0$ and $\alpha(q, \gamma, \beta)>0$ such that

$$
\lambda_{k+1}^{h}-\lambda_{k}^{h} \geq C_{3} k, \forall k \in \llbracket 1, \alpha N^{2 / 5} \rrbracket .
$$


Proof. Let $k \in \llbracket 1, N \rrbracket$. We apply Lemma 3.2 with $u^{h}:=\phi_{k}^{h}=\left(\phi_{p, k}^{h}\right)_{p \in \llbracket 1, N \rrbracket}$, $\lambda=\lambda_{k}^{h}$ and $f^{h}=0$. The vectors $U_{j}^{h}$ are then denoted by $U_{j, k}^{h}$ to keep track of the dependence on $k$. Moreover, with those notations, the quantity $\mathcal{R}_{k}^{h}$ defined in (19) can be expressed as

$$
\mathcal{R}_{k}^{h}=\inf _{i, j \in \llbracket 1, N+1 \rrbracket} \frac{\left\|U_{i, k}^{h}\right\|^{2}}{\left\|U_{j, k}^{h}\right\|^{2}} .
$$

Therefore, to obtain the announced lower bound, we only have to obtain a bound of $\left\|U_{j, k}^{h}\right\|$ by $\left\|U_{i, k}^{h}\right\|$ for any two indices $i, j$.

From the estimate (23) and the standard inequality $1+t \leq e^{t}, \forall t \geq 0$, we find the upper-bound

$$
\left\|\mathrm{I}+h_{j} M_{j, k}^{h}+h_{j} Q_{j, k}^{h}\right\| \leq \exp \left(h_{j} C \sqrt{\lambda_{k}^{h}}\right), \quad \forall j \in \llbracket 1, N \rrbracket .
$$

Direct computations, similar to the ones in the proof Lemma 3.2, also show that

$$
\left\|\left(\mathrm{I}+h_{j} M_{j, k}^{h}+h_{j} Q_{j, k}^{h}\right)^{-1}\right\| \leq \exp \left(h_{j} C \sqrt{\lambda_{k}^{h}}\right), \forall j \in \llbracket 1, N \rrbracket .
$$

Then, using (21) with $f^{h}=0$, we immediately obtain

$$
\exp \left(-C \sqrt{\lambda_{k}^{h}}\right)\left\|U_{j, k}^{h}\right\| \leq\left\|U_{i, k}^{h}\right\|, \forall i, j \in \llbracket 1, N+1 \rrbracket .
$$

By (25) and Lemma 3.1, the claim follows.

We now prove the gap property for a part of the spectrum. To this end, we will use Proposition 3.1 below. Take $k$ a positive integer such that $k \leq \alpha N^{2 / 5}$, where $\alpha(q, \gamma, \beta)>0$ will be chosen later on. Then, $\exists \bar{C}(q, \gamma, \beta)>0$, such that

$$
\begin{aligned}
\lambda_{k+1}^{h}-\lambda_{k}^{h} & \geq \lambda_{k+1}-\lambda_{k}-\left|\lambda_{k+1}^{h}-\lambda_{k+1}\right|-\left|\lambda_{k}^{h}-\lambda_{k}\right| \\
& \geq \bar{C} k-C h^{2} \lambda_{k}^{3} \\
& \geq \bar{C} k-C h^{2} k^{6},
\end{aligned}
$$

and the last inequality comes from (2). Now since

$$
h^{2} k^{6}=\left(h^{2} k^{5}\right) k \leq\left(h^{2} \alpha^{5} N^{2}\right) k \leq \Theta_{h}^{2} \alpha^{5} k \leq \beta^{2} \alpha^{5} k,
$$

we can write

$$
\lambda_{k+1}^{h}-\lambda_{k}^{h} \geq \bar{C} k-C \beta^{2} \alpha^{5} k .
$$

Now take $\alpha$ such that $\bar{C} \geq 2 C \beta^{2} \alpha^{5}$ to get

$$
\lambda_{k+1}^{h}-\lambda_{k}^{h} \geq \frac{\bar{C}}{2} k .
$$

This concludes the proof.

We have used above the following somehow standard numerical analysis result, whose proof is omitted. It can be obtained by similar techniques as in [6] and [20] and using the convergence analysis of finite difference methods on general grids given in [5]. 
Proposition 3.1. Assume that $q$ and $\gamma$ satisfy $\left(\mathrm{H}_{2}\right)$. For any $\beta>0$, there exists $C(\gamma, q, \beta)>0$, such that for any mesh satisfying $\Theta_{h} \leq \beta$, we have

$$
\left|\lambda_{k}^{h}-\lambda_{k}\right| \leq C h^{2} \lambda_{k}^{3}, \forall k \in \llbracket 1, N \rrbracket .
$$

3.3. Estimates for of a uniform grid, cases (S2) and (S3). In the previous subsection, the exponentially small lower bound comes from the crude estimate of the norm of the propagation matrix I $+h_{j} M_{j, k}^{h}$ by $1+C h_{j} \sqrt{\lambda_{k}^{h}}$. Besides, as noticed in Remark 3.1, the counter-part in the continuous case of this propagation matrix is $\exp \left(\int_{x_{j}}^{x_{j+1}} M(s) d s\right)$ which is a unitary matrix (see the proof of Lemma 2.3).

Thus, our objective is now to improve those estimates in the case of a uniform mesh. To this end, we introduce the discrete resolvant $S_{i \leftarrow j, k}^{h}$ defined by

$$
S_{i \leftarrow j, k}^{h}:=\left\{\begin{aligned}
\left(\mathrm{I}+h M_{i-1, k}^{h}\right)\left(\mathrm{I}+h M_{i-2, k}^{h}\right) \cdots\left(\mathrm{I}+h M_{j, k}^{h}\right) & \text { for } i>j \\
\mathrm{I} & \text { for } i=j \\
\left(S_{j \leftarrow i, k}^{h}\right)^{-1} & \text { for } i<j
\end{aligned}\right.
$$

and for which we will be able to prove sharper estimates. Observe that each matrix $\mathrm{I}+h M_{i, k}^{h}$ is invertible since its determinant is equal to one for a uniform grid (see $(22))$.

LEMma 3.4. With the same notations as in Lemma 3.2, there exists $C(q, \gamma)>0$, and $h_{0}(q, \gamma)>0$, such that for any $h<h_{0}$, and any $i, j \in \llbracket 1, N+1 \rrbracket$,

$$
\begin{aligned}
\left\|U_{i, k}^{h}\right\| \leq C \exp \left(C \sum_{p \in \llbracket i, j \rrbracket \backslash\{i\}} h\left\|S_{i \leftarrow p+1, k}^{h}\right\|\left\|Q_{p, k}^{h}\right\|\right) \\
\quad \times\left[\left\|S_{i \leftarrow j, k}^{h}\right\|\left\|U_{j, k}^{h}\right\|+\sum_{p \in \llbracket i, j \rrbracket \backslash\{i\}} h\left\|S_{i \leftarrow p+1, k}^{h}\right\|\left\|F_{p, k}^{h}\right\|\right] .
\end{aligned}
$$

Proof. Let $i, j \in \llbracket 1, N+1 \rrbracket$. From (21), discrete Duhamel formula gives

$$
\begin{aligned}
& \text { for } i>j: \quad U_{i, k}^{h}=S_{i \leftarrow j, k}^{h} U_{j, k}^{h}+h \sum_{p=j}^{i-1} S_{i \leftarrow p+1, k}^{h}\left(Q_{p, k}^{h} U_{p, k}^{h}+F_{p, k}^{h}\right), \\
& \text { for } i<j: \quad U_{i, k}^{h}=S_{i \leftarrow j, k}^{h} U_{j, k}^{h}-h \sum_{p=i}^{j-1} S_{i \leftarrow p+1, k}^{h}\left(Q_{p, k}^{h} U_{p, k}^{h}+F_{p, k}^{h}\right) .
\end{aligned}
$$

So for any $i, j \in \llbracket 1, N+1 \rrbracket$,

$$
\left\|U_{i, k}^{h}\right\| \leq\left\|S_{i \leftarrow j, k}^{h}\right\|\left\|U_{j, k}^{h}\right\|+h \sum_{p \in \llbracket i, j \rrbracket}\left\|S_{i \leftarrow p+1, k}^{h}\right\|\left(\left\|Q_{p, k}^{h}\right\|\left\|U_{p, k}^{h}\right\|+\left\|F_{p, k}^{h}\right\|\right) .
$$

Using the bound (23) on $\left\|Q_{i, k}^{h}\right\|$,

$$
\begin{aligned}
\left\|U_{i, k}^{h}\right\|\left(1-C h\left\|S_{i \leftarrow i+1, k}^{h}\right\|\right) \leq & \left\|S_{i \leftarrow j, k}^{h}\right\|\left\|U_{j, k}^{h}\right\| \\
& +h \sum_{p \in \llbracket i, j \rrbracket \backslash\{i\}}\left\|S_{i \leftarrow p+1, k}^{h}\right\|\left(\left\|Q_{p, k}^{h}\right\|\left\|U_{p, k}^{h}\right\|+\left\|F_{p, k}^{h}\right\|\right) .
\end{aligned}
$$


SPECTRAL ANALYSIS OF DISCRETE ELLIPTIC OPERATORS AND APPLICATIONS 21

Note also that $\left\|S_{i \leftarrow i+1, k}^{h}\right\|=\left\|\mathrm{I}+h M_{i, k}^{h}\right\| \leq 1+C h \sqrt{\lambda_{k}^{h}}$. Thus, by Lemma 3.3 and Remark 1.3 (remember that the mesh is assumed to be uniform), for $h$ small enough and some $C(q, \gamma)>0$, we have

$$
\left\|U_{i, k}^{h}\right\| \leq C\left\|S_{i \leftarrow j, k}^{h}\right\|\left\|U_{j, k}^{h}\right\|+C h \sum_{p \in \llbracket i, j \rrbracket \backslash\{i\}}\left\|S_{i \leftarrow p+1, k}^{h}\right\|\left(\left\|Q_{p, k}^{h}\right\|\left\|U_{p, k}^{h}\right\|+\left\|F_{p, k}^{h}\right\|\right) .
$$

We conclude with discrete Gronwall's Lemma.

We recall that in the continuous case we had the fundamental property that $\left\|S\left(x_{0}, x\right)\right\|=1$. Here we will not obtain that the discrete resolvant $S_{j \leftarrow i, k}^{h}$ is unitary but we are able to produce uniform bounds on this object at least for a well chosen portion of the discrete spectrum.

Suppose that $q$ and $\gamma$ satisfy assumption $\left(\mathrm{H}_{0}\right)$. For any $\varepsilon \geq 0$, we define the following integer

$$
k_{\max , \varepsilon}^{h}:=\max \left\{k \in \llbracket 1, N \rrbracket ; \lambda_{k}^{h}<\frac{4}{h^{2}} \gamma_{\min }(1-\varepsilon)\right\} .
$$

Proposition 3.2. Let $q$ and $\gamma$ satisfying $\left(\mathrm{H}_{0}\right)$. There exists $h_{0}(q, \gamma)>0$ and $C(q, \gamma)>0$ such that, for any $\varepsilon>0$ and any uniform mesh of size $h<h_{0}$, we have the estimate

$$
\left\|S_{i \leftarrow j, k}^{h}\right\| \leq C \exp \left(\frac{C}{\varepsilon}\right), \forall i, j \in \llbracket 1, N+1 \rrbracket, \quad \forall k \in \llbracket 1, k_{\max , \varepsilon}^{h} \rrbracket .
$$

If we additionally assume that the diffusion coefficient $\gamma$ is constant, then the following estimate holds for the whole spectrum of $\mathcal{A}^{h}$, for any $h<h_{0}$

$$
\left\|S_{i \leftarrow j, k}^{h}\right\| \leq \frac{C}{\sqrt{1-\frac{h^{2}}{4 \gamma}\left(\lambda_{k}^{h}-\|q\|_{L^{\infty}}\right)}}, \forall i, j \in \llbracket 1, N+1 \rrbracket, \quad \forall k \in \llbracket 1, N \rrbracket .
$$

Note that, by Lemma 3.3 and Remark 1.3, there exists a $\alpha(q, \gamma)>0$ such that

$$
k_{\max , \varepsilon}^{h} \geq \alpha N \sqrt{1-\varepsilon}, \quad \forall h>0 .
$$

Thus, inequality (30) in the previous proposition gives actually a uniform estimate of $S_{\bullet \leftarrow \bullet, k}^{h}$ for (at least) a constant portion of the eigenelements of the discrete operator $\mathcal{A}^{h}$.

Proof. Since $S_{i \leftarrow j, k}^{h} U_{j, k}^{h}=U_{i, k}^{h}$, the study of the norm of $S_{i \leftarrow j, k}^{h}$ amounts in obtaining a bound on $U_{i, k}^{h}$ in function of $U_{j, k}^{h}$, for any $i, j$, where $U_{\bullet, k}^{h}$ is any solution of the recurrence relation

$$
U_{i+1, k}^{h}=\left(\mathrm{I}+h M_{i, k}^{h}\right) U_{i, k}^{h}, \quad \forall i \in \llbracket 1, N+1 \rrbracket .
$$

To simplify the notation in this proof, we shall sometimes drop the indices $h$ and $k$ but we keep in mind that the condition $k \leq k_{\max , \varepsilon}^{h}$ implies that the eigenvalue $\lambda=\lambda_{k}^{h}$ we are considering satisfies the bound

$$
\lambda<\frac{4}{h^{2}} \gamma_{\min }(1-\varepsilon) .
$$


If we denote by $x_{i}, y_{i}$ the two components of $U_{i}$, according to (22), relation (32) reads

$$
\left\{\begin{array}{l}
x_{i+1}=x_{i}\left(1-\frac{h^{2} \lambda}{\gamma_{i+1 / 2}}\right)+y_{i} h \sqrt{\frac{\lambda}{\gamma_{i+1 / 2}}} \\
y_{i+1}=-x_{i} h \sqrt{\frac{\lambda}{\gamma_{i+1 / 2}}}+y_{i} .
\end{array}\right.
$$

The key-point is now to introduce the following quantity

$$
H_{i}:=x_{i}^{2}+y_{i}^{2}-h \sqrt{\frac{\lambda}{\gamma_{i+1 / 2}}} x_{i} y_{i} .
$$

As we will see below, this quantity is conserved in the recurrence process $\left(H_{i+1}=H_{i}\right)$, when $\gamma$ is constant. However, in the general case, this conservation does not hold anymore and we need sharper estimates.

After some tedious but straightfoward computations, we end up with the following identity

$$
\begin{aligned}
H_{i+1} & =H_{i}+\left(\frac{1}{\sqrt{\gamma_{i+1 / 2}}}-\frac{1}{\sqrt{\gamma_{i+3 / 2}}}\right) \\
& \times\left[x_{i}^{2} \frac{h^{2} \lambda}{\sqrt{\gamma_{i+1 / 2}}}\left(\frac{h^{2} \lambda}{\gamma_{i+1 / 2}}-1\right)+y_{i}^{2} \frac{h^{2} \lambda}{\sqrt{\gamma_{i+1 / 2}}}+x_{i} y_{i} h \sqrt{\lambda}\left(1-\frac{2 h^{2} \lambda}{\gamma_{i+1 / 2}}\right)\right] .
\end{aligned}
$$

Using the regularity assumptions on $\gamma$ and the mean-value theorem, we deduce that for some $C(\gamma)>0$

$$
\left|H_{i+1}\right| \leq\left|H_{i}\right|+h C(\gamma)\left[\frac{2 h^{2} \lambda}{\sqrt{\gamma_{\min }}}\left(\frac{h^{2} \lambda}{\gamma_{\min }}+1\right)+\frac{h \sqrt{\lambda}}{2}\left(1+\frac{2 h^{2} \lambda}{\gamma_{\min }}\right)\right]\left(x_{i}^{2}+y_{i}^{2}\right) .
$$

Moreover, we know from Lemma 3.3 that the quantity $h^{2} \lambda$ is uniformly bounded by some constant depending only on $q$ and $\gamma$. It finally follows that, for some $C(q, \gamma)>0$, we have

$$
\left|H_{i+1}\right| \leq\left|H_{i}\right|+h C\left(x_{i}^{2}+y_{i}^{2}\right), \quad \forall i \in \llbracket 1, N \rrbracket .
$$

Besides, from definition (35) of $H_{i}$, we easily get that

$$
\left(x_{i}^{2}+y_{i}^{2}\right)\left(1-\frac{h}{2} \sqrt{\frac{\lambda}{\gamma_{i+1 / 2}}}\right) \leq H_{i} \leq\left(x_{i}^{2}+y_{i}^{2}\right)\left(1+\frac{h}{2} \sqrt{\frac{\lambda}{\gamma_{i+1 / 2}}}\right) .
$$

Using again that $h^{2} \lambda$ is uniformly bounded, the right-hand side term can be replaced by some $C\left(x_{i}^{2}+y_{i}^{2}\right)$. However, the factor in front of $\left(x_{i}^{2}+y_{i}^{2}\right)$ in the left-hand side may become negative for large eigenvalues. That is exactly the reason why the condition (33) on $\lambda$ (which is nothing but the condition on $k$ in the statement of the proposition) enters into the analysis. Indeed, if we assume (33), the above inequalities become

$$
\frac{\varepsilon}{2}\left(x_{i}^{2}+y_{i}^{2}\right) \leq H_{i} \leq C(q, \gamma)\left(x_{i}^{2}+y_{i}^{2}\right) .
$$


SPECTRAL ANALYSIS OF DISCRETE ELLIPTIC OPERATORS AND APPLICATIONS 23

From (37), we can thus infer that

$$
\left|H_{i+1}\right| \leq\left|H_{i}\right|+\frac{C}{\varepsilon} h\left|H_{i}\right| \leq \exp (h C / \varepsilon)\left|H_{i}\right|, \quad \forall i \in \llbracket 1, N \rrbracket,
$$

and finally, for any $i \geq j$, we get

$$
\left|H_{i}\right| \leq \exp ((i-j) h C / \varepsilon)\left|H_{j}\right| \leq \exp (C / \varepsilon)\left|H_{j}\right| .
$$

Using again (39), we deduce that

$$
\left(x_{i}^{2}+y_{i}^{2}\right) \leq \frac{C}{\varepsilon} \exp \left(\frac{C}{\varepsilon}\right)\left(x_{j}^{2}+y_{j}^{2}\right) .
$$

This exactly shows (30) in the case where $i \geq j$.

To prove the same equality for $j \geq i$, we come back to the equality (36) to get

$$
\left|H_{i}\right| \leq\left|H_{i+1}\right|+C h\left(x_{i}^{2}+y_{i}^{2}\right) .
$$

Then, from (34), we find

$$
\left(\begin{array}{l}
x_{i} \\
y_{i}
\end{array}\right)=\left(\begin{array}{cc}
1 & -h \sqrt{\frac{\lambda}{\gamma_{i+1 / 2}}} \\
h \sqrt{\frac{\lambda}{\gamma_{i+1 / 2}}} & 1-h^{2} \frac{\lambda}{\gamma_{i+1 / 2}}
\end{array}\right)\left(\begin{array}{l}
x_{i+1} \\
y_{i+1}
\end{array}\right)
$$

and using again that $h^{2} \lambda$ is uniformly bounded we obtain that, for some $C(q, \gamma)>0$,

$$
x_{i}^{2}+y_{i}^{2} \leq C\left(x_{i+1}^{2}+y_{i+1}^{2}\right), \forall i \in \llbracket 1, N \rrbracket .
$$

Hence, (40) becomes

$$
\left|H_{i}\right| \leq\left|H_{i+1}\right|+C h\left(x_{i+1}^{2}+y_{i+1}^{2}\right) \leq\left|H_{i+1}\right|\left(1+\frac{C}{\varepsilon} h\right),
$$

and we can argue as before to prove our claim, when $j \geq i$.

Let us consider now the case where $\gamma$ is constant.

- We first assume that the eigenvalue satisfies

$$
\frac{h^{2} \lambda}{4 \gamma} \leq 1-\frac{h^{2}}{\gamma} .
$$

Since $\gamma$ is constant, we have $H_{i}=H_{j}$ for any $i, j$ (see (36)) and from (38) we have

$$
\left(1-\frac{h}{2} \sqrt{\frac{\lambda}{\gamma}}\right)\left(x_{i}^{2}+y_{i}^{2}\right) \leq H_{i}=H_{j} \leq C\left(x_{j}^{2}+y_{j}^{2}\right) .
$$

By (41), we observe that the first factor in the left-hand side is positive, so that we can write

$$
x_{i}^{2}+y_{i}^{2} \leq \frac{C}{1-\frac{h}{2} \sqrt{\frac{\lambda}{\gamma}}}\left(x_{j}^{2}+y_{j}^{2}\right), \forall i, j \in \llbracket 1, N+1 \rrbracket,
$$

and the claim follows since, using again (41), we have

$$
\frac{1}{1-\frac{h}{2} \sqrt{\frac{\lambda}{\gamma}}}=\frac{1+\frac{h}{2} \sqrt{\frac{\lambda}{\gamma}}}{1-\frac{h^{2} \lambda}{4 \gamma}} \leq \frac{C}{1-\frac{h^{2} \lambda}{4 \gamma}} \leq \frac{C\left(1+\frac{\|q\|_{\infty}}{4}\right)}{1-\frac{h^{2}\left(\lambda-\|q\|_{L} \infty\right)}{4 \gamma}} .
$$


- Assume now that the eigenvalue does not satisfy (41). By Lemma 3.3 and Remark 1.3, this implies that

$$
1-\frac{h^{2}}{\gamma}<\frac{h^{2} \lambda}{4 \gamma} \leq 1-h^{2}+\frac{h^{2}\|q\|_{L^{\infty}}}{4 \gamma}
$$

We set $\widetilde{\lambda}=\lambda-\|q\|_{L^{\infty}}$. We denote by $\widetilde{M}_{\bullet, k}^{h}$ and $\tilde{S}_{\bullet \leftarrow \bullet, k}^{h}$ the same matrices as $M_{\bullet, k}^{h}$ and $S_{\bullet \leftarrow \bullet, k}^{h}$ with $\lambda$ replaced by $\widetilde{\lambda}$. An explicit computation shows that, for $h$ small enough and using the left-hand side inequality of (42), we have

$$
\left\|M_{i, k}^{h}-\widetilde{M}_{i, k}^{h}\right\| \leq C \frac{|\lambda-\widetilde{\lambda}|}{\sqrt{\lambda}+\sqrt{\tilde{\lambda}}} \leq C h, \quad \forall i \in \llbracket 1, N \rrbracket .
$$

Since, by construction, $\tilde{\lambda}$ satisfies the assumption (41), we have that

$$
\left\|\tilde{S}_{i \leftarrow j, k}^{h}\right\| \leq \frac{C}{\sqrt{1-\frac{h^{2} \widetilde{\lambda}}{4 \gamma}}}, \forall i, j \in \llbracket 1, N+1 \rrbracket .
$$

With those notations, the recurrence relation (32) can be written as follows

$$
U_{i+1, k}^{h}=\left(\mathrm{I}+h \widetilde{M}_{i, k}^{h}\right) U_{i, k}^{h}+h\left(M_{i, k}^{h}-\widetilde{M}_{i, k}^{h}\right) U_{i, k}^{h}, \quad \forall i \in \llbracket 1, N+1 \rrbracket .
$$

This formula has the same form as (21) with $F_{i, k}^{h}=0$ and $Q_{i, k}^{h}=M_{i, k}^{h}-\widetilde{M}_{i, k}^{h}$ so that we can use the inequality (26) in this context, as well as (43) and (44), to finally get

$$
\left\|U_{i, k}^{h}\right\| \leq \frac{C}{\sqrt{1-\frac{h^{2} \tilde{\lambda}}{4 \gamma}}} \exp \left(\frac{C h}{\sqrt{1-\frac{h^{2} \tilde{\lambda}}{4 \gamma}}}\right)\left\|U_{j, k}^{h}\right\|, \forall i, j \in \llbracket 1, N+1 \rrbracket .
$$

Finally, since $\tilde{\lambda}$ satisfies (41), we have $\sqrt{1-\frac{h^{2} \widetilde{\lambda}}{4 \gamma}} \geq \frac{h}{\sqrt{\gamma}}$ which proves that the exponential factor in the estimate above is actually uniformly bounded. It follows that

$$
\left\|U_{i, k}^{h}\right\| \leq \frac{C}{\sqrt{1-\frac{h^{2} \widetilde{\lambda}}{4 \gamma}}}\left\|U_{j, k}^{h}\right\|, \forall i, j \in \llbracket 1, N+1 \rrbracket,
$$

which exactly proves the claimed estimate. (S2).

We can now state and prove the following discrete spectral estimates in the setting

THEOREM 3.2 (The case of a uniform grid). Let $\gamma$ and $q$ satisfying $\left(\mathrm{H}_{0}\right), \omega \subset \Omega$ a non empty open subset of $\Omega$ and $\varepsilon>0$. There exists $h_{1}(q, \gamma)>0, h_{2}(q, \gamma, \omega)>0$ and $C_{1}(q, \gamma, \varepsilon)>0, C_{2}(q, \gamma, \omega, \varepsilon)>0$ so that for any uniform mesh of size $h$, we have

$$
\begin{gathered}
\frac{1}{\sqrt{\lambda_{k}^{h}}}\left|\partial_{b} \phi_{k}^{h}\right| \geq C_{1}, \forall k \in \llbracket 1, k_{\max , \varepsilon}^{h} \rrbracket, \forall b \in\{l, r\}, \forall h<h_{1}, \\
\left\|\phi_{k}^{h}\right\|_{L^{2}\left(\omega_{h}\right)}^{2} \geq C_{2}, \quad \forall k \in \llbracket 1, k_{\max , \varepsilon}^{h} \rrbracket, \forall h<h_{2},
\end{gathered}
$$

and

$$
\lambda_{k+1}^{h}-\lambda_{k}^{h} \geq C_{1} \sqrt{\lambda_{k+1}^{h}}, \quad \forall k \in \llbracket 1, k_{\max , \varepsilon}^{h}-1 \rrbracket, \forall h<h_{1} .
$$


Proof. We apply Lemma 3.2 with $u^{h}=\phi_{k}^{h}, \lambda=\lambda_{k}^{h}$ and $f^{h}=0$. From Lemma 3.4 and inequality (30), we get for $i, j \in \llbracket 1, N+1 \rrbracket$,

$$
\left\|U_{i, k}^{h}\right\| \leq C \exp \left(\exp (C / \varepsilon) C \sum_{p \in \llbracket i, j \rrbracket \backslash\{i\}} h\left\|Q_{p, k}^{h}\right\|\right) \times \exp (C / \varepsilon)\left\|U_{j, k}^{h}\right\| .
$$

According to (23), we have a uniform bound $\left\|Q_{p, k}^{h}\right\| \leq C$, so that we finally obtain

$$
\left\|U_{i, k}^{h}\right\| \leq C(q, \gamma, \varepsilon)\left\|U_{j, k}^{h}\right\|, \quad \forall i, j \in \llbracket 1, N+1 \rrbracket .
$$

By (25), we just have shown that

$$
\mathcal{R}_{k}^{h} \geq \frac{1}{C(q, \gamma, \varepsilon)^{2}},
$$

which gives the first two lower bounds by virtue of Lemma 3.1.

Let us now estimate the difference $\lambda_{k+1}^{h}-\lambda_{k}^{h}$ for any $k \in \llbracket 1, k_{\max , \varepsilon}^{h}-1 \rrbracket$. We follow the same lines as in the proof in the continuous case in Section 2. We set $u^{h}=\left(\partial_{r} \phi_{k}^{h}\right) \phi_{k+1}^{h}-\left(\partial_{r} \phi_{k+1}^{h}\right) \phi_{k}^{h}$ and $f^{h}=\left(\lambda_{k+1}^{h}-\lambda_{k}^{h}\right)\left(\partial_{r} \phi_{k+1}^{h}\right) \phi_{k}^{h}$, so that (20) is satisfied with $\lambda=\lambda_{k+1}^{h}$. We use the same notation as in Lemma 3.2, and we observe that

$$
U_{N+1}^{h}=\left(\begin{array}{c}
u_{N+1}^{h} \\
\sqrt{\frac{\gamma_{N+1 / 2}}{\lambda_{k+1}^{h}}} \partial_{r} u^{h}
\end{array}\right)=\left(\begin{array}{l}
0 \\
0
\end{array}\right),
$$

since $\phi_{k+1}^{h}$ and $\phi_{k}^{h}$ satisfy the homogeneous boundary conditions and moreover, by construction, $\partial_{r} u^{h}=0$.

We can then apply Lemma 3.4, with $j=N+1$, and the estimates (23) and (30) to get

$$
\left\|U_{i}^{h}\right\| \leq C(q, \gamma, \varepsilon) \sum_{p \in \llbracket i, j \rrbracket \backslash\{i\}} h\left\|F_{p}^{h}\right\|, \forall i \in \llbracket 1, N+1 \rrbracket .
$$

By definition of $F^{h}$ and $f^{h}$, we have

$$
\left\|F_{p}^{h}\right\| \leq \frac{C(\gamma)}{\sqrt{\lambda_{k+1}^{h}}}\left(\lambda_{k+1}^{h}-\lambda_{k}^{h}\right)\left|\partial_{r} \phi_{k+1}^{h} \| \phi_{p, k}^{h}\right|, \quad \forall p \in \llbracket 1, N \rrbracket .
$$

Since $\phi_{k}^{h}$ is normalized in $L^{2}\left(\Omega_{h}\right)$ we conclude that

$$
\left\|U_{i}^{h}\right\| \leq \frac{C(q, \gamma, \varepsilon)}{\sqrt{\lambda_{k+1}^{h}}}\left(\lambda_{k+1}^{h}-\lambda_{k}^{h}\right)\left|\partial_{r} \phi_{k+1}^{h}\right|
$$

By definition of $U_{i}^{h}$, we have $\left|u_{i}^{h}\right| \leq\left\|U_{i}^{h}\right\|$ for any $i \in \llbracket 1, N \rrbracket$ and thus, the discrete $L^{2}$ norm of $u^{h}$ is estimated by

$$
\left\|u^{h}\right\|_{L^{2}\left(\Omega_{h}\right)}^{2} \leq \frac{C(q, \gamma, \varepsilon)}{\lambda_{k+1}^{h}}\left|\lambda_{k+1}^{h}-\lambda_{k}^{h}\right|^{2}\left|\partial_{r} \phi_{k+1}^{h}\right|^{2} .
$$

By definition of $u^{h}$ and since $\phi_{k+1}^{h}$ and $\phi_{k}^{h}$ are orthonormal we have

$$
\left\|u^{h}\right\|_{L^{2}\left(\Omega_{h}\right)}^{2}=\left(\partial_{r} \phi_{k+1}^{h}\right)^{2}+\left(\partial_{r} \phi_{k}^{h}\right)^{2} .
$$


Combining the two previous inequalities (and using that $\partial_{r} \phi_{k+1}^{h} \neq 0$ ) we finally get

$$
1 \leq \frac{C(q, \gamma, \varepsilon)}{\lambda_{k+1}^{h}}\left|\lambda_{k+1}^{h}-\lambda_{k}^{h}\right|^{2}
$$

which gives the claim.

Finally, in the case of a constant diffusion coefficient and uniform grids we obtain a stronger result with precise lower bounds for all the eigenfunctions of $\mathcal{A}^{h}$.

THEOREM 3.3 (Uniform grid - constant diffusion). Let $q$ be a continuous function on $\bar{\Omega}$ and assume that $\gamma$ is a constant function. Let $\omega \subset \Omega$ be a non-empty open subset of $\Omega$. There exist $h_{1}(q, \gamma)>0, h_{2}(q, \gamma, \omega)>0$ sufficiently small and $C_{1}(q, \gamma)>0$, $C_{2}(q, \gamma, \omega)>0$ such that for any uniform mesh of size $h$, we have for $h<h_{1}$,

$$
\frac{1}{\sqrt{\lambda_{k}^{h}}}\left|\partial_{b} \phi_{k}^{h}\right| \geq C_{1} \sqrt{1-\frac{h^{2}\left(\lambda_{k}^{h}-\|q\|_{L^{\infty}}\right)}{4 \gamma}}, \forall k \in \llbracket 1, N \rrbracket, \forall b \in\{l, r\},
$$

and

$$
\left\|\phi_{k}^{h}\right\|_{L^{2}\left(\omega_{h}\right)}^{2} \geq C_{2}\left(1-\frac{h^{2}\left(\lambda_{k}^{h}-\|q\|_{\left.L^{\infty}\right)}\right.}{4 \gamma}\right), \forall k \in \llbracket 1, N \rrbracket,
$$

for $h<h_{2}$. Moreover,

$$
\lambda_{k+1}^{h}-\lambda_{k}^{h} \geq C_{1} \sqrt{\lambda_{k+1}^{h}} \sqrt{1-\frac{h^{2}\left(\lambda_{k+1}^{h}-\|q\|_{L^{\infty}}\right)}{4 \gamma}}, \forall k \in \llbracket 1, N-1 \rrbracket,
$$

as soon as $h<h_{1}$.

Proof. We shall use the notation $\widetilde{\lambda}_{k}^{h}=\lambda_{k}^{h}-\|q\|_{L^{\infty}}$. We follow exactly the same lines as in the proof of the previous theorem, except that the estimate (30) is now improved into (31). It follows that, for any $i, j \in \llbracket 1, N+1 \rrbracket$ we have

$$
\left\|U_{i, k}^{h}\right\| \leq \frac{C}{\sqrt{1-\frac{h^{2}}{4} \frac{\widetilde{\frac{\lambda}{k}}_{\gamma}^{h}}{\gamma}}} \exp \left(\frac{C}{\sqrt{1-\frac{h^{2}}{4} \frac{\widetilde{\lambda}_{k}^{h}}{\gamma}}} \sum_{p \in \llbracket i, j \rrbracket \backslash\{i\}} h\left\|Q_{p, k}^{h}\right\|\right)\left\|U_{j, k}^{h}\right\| .
$$

According to (24), we have the bound $\left\|Q_{p, k}^{h}\right\| \leq C\left(h+\frac{1}{\sqrt{\lambda_{k}^{h}}}\right)$, for any $p$ and thus

$$
\left\|U_{i, k}^{h}\right\| \leq \frac{C}{\sqrt{1-\frac{h^{2} \widetilde{\lambda}_{k}^{h}}{4 \gamma}}} \exp \left(C \frac{h+\frac{1}{\sqrt{\lambda_{k}^{h}}}}{\sqrt{1-\frac{h^{2} \widetilde{\lambda}_{k}^{h}}{4 \gamma}}}\right)\left\|U_{j, k}^{h}\right\| .
$$

It remains to prove that the exponential factor is actually uniformly bounded.

- Assume first that $h^{2} \widetilde{\lambda}_{k}^{h} \leq \gamma$, so that we have

$$
\sqrt{1-\frac{h^{2} \widetilde{\lambda}_{k}^{h}}{4 \gamma}} \geq \sqrt{3 / 4}
$$

and the exponential term above is clearly bounded. 
- Assume now that $\gamma \leq h^{2} \widetilde{\lambda}_{k}^{h}$. We have already seen that we also have $h^{2} \widetilde{\lambda}_{k}^{h} \leq$ $4 \gamma\left(1-h^{2}\right)$ so that

$$
\sqrt{1-\frac{h^{2} \widetilde{\lambda}_{k}^{h}}{4 \gamma}} \geq h
$$

and then

$$
\frac{h+\frac{1}{\sqrt{\lambda_{k}^{h}}}}{\sqrt{1-\frac{h^{2} \widetilde{\lambda}_{k}^{h}}{4 \gamma}}} \leq\left(1+\frac{1}{h \sqrt{\lambda_{k}^{h}}}\right) \leq\left(1+\frac{1}{h \sqrt{\tilde{\lambda}_{k}^{h}}}\right) \leq\left(1+\frac{1}{\sqrt{\gamma}}\right),
$$

and we reach the same conclusion.

To sum up, we have just proved that

$$
\left\|U_{i, k}^{h}\right\| \leq \frac{C}{\sqrt{1-\frac{h^{2} \widetilde{\lambda}_{k}^{h}}{4 \gamma}}}\left\|U_{j, k}^{h}\right\|,
$$

which implies, with (25), that

$$
\mathcal{R}_{k}^{h} \geq C\left(1-\frac{h^{2} \widetilde{\lambda}_{k}^{h}}{4 \gamma}\right), \quad \forall k \in \llbracket 1, N \rrbracket .
$$

With Lemma 3.1 the first two inequalities are proved.

As in the proof of Theorem 3.2, the gap property is proved by defining $u^{h}=$ $\left(\partial_{r} \phi_{k}^{h}\right) \phi_{k+1}^{h}-\left(\partial_{r} \phi_{k+1}^{h}\right) \phi_{k}^{h}$ and $f^{h}=\left(\lambda_{k+1}^{h}-\lambda_{k}^{h}\right)\left(\partial_{r} \phi_{k+1}^{h}\right) \phi_{k}^{h}$, and using the uniform estimate above on $\exp \left(C \frac{h+\frac{1}{\sqrt{\lambda_{k}^{h}}}}{\sqrt{1-\frac{h^{2} \hat{\lambda}_{k}^{h}}{4 \gamma}}}\right)$.

A careful inspection of the arguments in the above proof shows that we have the estimate

$$
\lambda_{k}^{h}\left(1-\frac{h^{2}}{4 \gamma}\left(\lambda_{k}^{h}-\|q\|_{\infty}\right)\right) \geq C(q, \gamma), \quad \forall k \in \llbracket 1, N \rrbracket .
$$

This immediately gives the following corollary

COROLlaRY 3.1. With the same assumptions and notations as in the previous theorem (the values of $C_{1}$ and $C_{2}$ being possibly different) we have

$$
\begin{gathered}
\left|\partial_{b} \phi_{k}^{h}\right| \geq C_{1}, \forall k \in \llbracket 1, N \rrbracket, \forall b \in\{l, r\}, \\
\lambda_{k+1}^{h}-\lambda_{k}^{h} \geq C_{1}, \quad \forall k \in \llbracket 1, N-1 \rrbracket,
\end{gathered}
$$

as soon as $h<h_{1}$, and

$$
\left\|\phi_{k}^{h}\right\|_{L^{2}\left(\omega_{h}\right)}^{2} \geq \frac{C_{2}}{\lambda_{k}^{h}}, \forall k \in \llbracket 1, N \rrbracket,
$$

as soon as $h<h_{2}$. 


\subsection{Proof of Lemma 3.1.}

Proof. We follow exactly the same arguments as the ones given in the proof of Lemma 2.1. For the sake of completeness, we provide here some technical precisions.

- Let us prove that

$$
\frac{1}{\lambda_{k}^{h}}\left|\partial_{b} \phi_{k}^{h}\right|^{2} \geq C \mathcal{R}_{k}^{h}, \forall b \in\{l, r\} .
$$

We recall, from (19), the following identity for $i, j \in \llbracket 1, N+1 \rrbracket$

$$
\mathcal{R}_{k}^{h}\left(\left|\phi_{i, k}^{h}\right|^{2}+\frac{\gamma_{i-1 / 2}}{\lambda_{k}^{h}}\left|\frac{\phi_{i, k}^{h}-\phi_{i-1, k}^{h}}{h_{i-1 / 2}}\right|^{2}\right) \leq\left|\phi_{j, k}^{h}\right|^{2}+\frac{\gamma_{j-1 / 2}}{\lambda_{k}^{h}}\left|\frac{\phi_{j, k}^{h}-\phi_{j-1, k}^{h}}{h_{j-1 / 2}}\right|^{2} .
$$

With $j=N+1$, using Dirichlet boundary condition in $x=1$ and summing the left hand side for $i$ from 1 to $N$, we get (46) with $b=r$. For the case $b=l$, take $j=1$ and again, sum with respect to the variable $i$ :

$$
\mathcal{R}_{k}^{h} \leq\left|\phi_{1, k}^{h}\right|^{2}+\frac{\gamma_{1 / 2}}{\lambda_{k}^{h}}\left|\frac{\phi_{1, k}^{h}-0}{h_{1 / 2}}\right|^{2}=\left(h_{1 / 2}^{2}+\frac{\gamma_{1 / 2}}{\lambda_{k}^{h}}\right)\left|\frac{\phi_{1, k}^{h}-0}{h_{1 / 2}}\right|^{2}
$$

According to Lemma $3.3, h_{1 / 2}^{2} \lambda_{k}^{h} \leq C(q, \gamma, \beta)$, so $\mathcal{R}_{k}^{h} \leq \frac{C(q, \gamma, \beta)}{\lambda_{k}^{h}}\left|\frac{\phi_{1, k}^{h}-0}{h_{1 / 2}}\right|^{2}$.

- Denote by $\omega_{j}^{h}, j \in\{1,2,3\}$ the set of mesh points which belong to $\omega_{j}$. In the discrete setting, hypothesis (15) is replaced by: suppose that there exists $x_{i_{1}}$ and $x_{i_{3}}$ two points of $\omega_{1}^{h}$ and $\omega_{3}^{h}$ respectively, such that

$$
\phi_{i_{1}, k}^{h}\left(\phi_{i_{1}, k}^{h}-\phi_{i_{1}-1, k}^{h}\right) \geq 0 \text { and } \phi_{i_{2}, k}^{h}\left(\phi_{i_{2}+1}^{h}-\phi_{i_{2}}^{h}\right) \leq 0 .
$$

Thus, when multiplying the equation $\mathcal{A}^{h} \phi_{k}^{h}=\lambda_{k}^{h} \phi_{k}^{h}$ by $\phi_{k}^{h}$ and summing between $i_{1}$ and $i_{3}$, the discrete integration by parts leads to boundary terms (which are the analogues of $\left[\gamma(x) \phi_{k}^{\prime}(x) \phi_{k}(x)\right]_{a_{k}}^{b k}$ ) that are again non positive thanks to (47).

- Remark 2.3 still holds in the discrete setting. Indeed, denote by $i_{0}$ the index of the first point of $\omega_{3}^{h}$ and suppose that for all indices $i \in\{1, \ldots, N\}$ such that $x_{i} \in \omega_{3}^{h}$, we have $\phi_{i, k}^{h}\left(\phi_{i+1, k}^{h}-\phi_{i, k}^{h}\right)>0$. If $\phi_{i_{0}, k}^{h}>0$ then $\left(\phi_{i, k}^{h}\right)_{i, x_{i} \in \omega_{3}^{h}}$ is increasing and positive, else if $\phi_{i_{0}, k}^{h}<0$ then $\left(\phi_{i, k}^{h}\right)_{i, x_{i} \in \omega_{3}^{h}}$ is decreasing and negative.

Moreover, given that for all $i \in\{1, \ldots, N\}$ such that $x_{i} \in \omega_{3}^{h}$,

$$
0<\phi_{i, k}^{h}\left(\phi_{i+1, k}^{h}-\phi_{i, k}^{h}\right)=\frac{1}{2}\left(\left(\phi_{i+1, k}^{h}\right)^{2}-\left(\phi_{i, k}^{h}\right)^{2}\right)-\frac{1}{2}\left[\left(\phi_{i+1, k}^{h}\right)-\left(\phi_{i, k}^{h}\right)\right]^{2},
$$

we conclude that $\left(\left|\phi_{i, k}^{h}\right|^{2}\right)_{i, x_{i} \in \omega_{3}^{h}}$ is increasing on $\omega_{3}^{h}$.

4. Numerical illustrations. In this section we provide a few numerical simulations that aim to illustrate our theoretical results on the discrete spectral properties of the operator $\mathcal{A}^{h}$ obtained in the previous section. We will consider the following two control/observation domains $\omega^{1}=(0,0.3)$ and $\omega^{2}=(0.7,1)$.

Let us introduce the following notations for any $K \in \llbracket 1, N \rrbracket$

$$
I_{l}^{h}(K):=\min _{k \leq K}\left|\partial_{l} \phi_{k}^{h}\right|, \quad I_{r}^{h}(K):=\min _{k \leq K}\left|\partial_{r} \phi_{k}^{h}\right|
$$




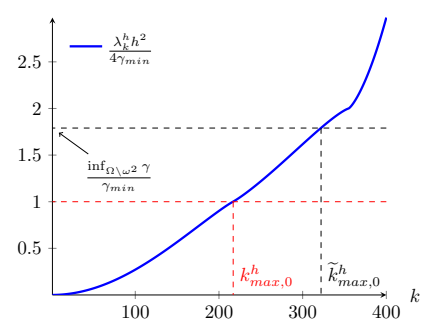

(a) Normalized discrete spectrum

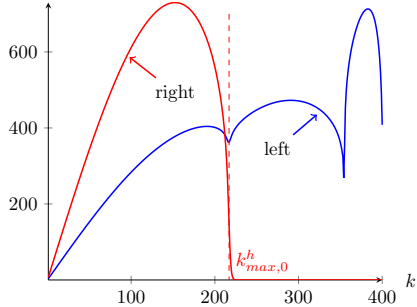

(b) Normal derivatives

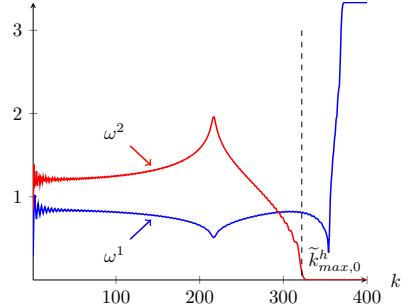

(c) $L^{2}\left(\omega_{h}^{i}\right)$ norms

Figure 2: Case $1-\mathrm{N}=400$

$$
I_{1}^{h}(K):=\min _{k \leq K} \frac{\left\|\phi_{k}^{h}\right\|_{L^{2}\left(\omega_{h}^{1}\right)}^{2}}{\left|\omega^{1}\right|}, \quad I_{2}^{h}(K):=\min _{k \leq K} \frac{\left\|\phi_{k}^{h}\right\|_{L^{2}\left(\omega_{h}^{2}\right)}^{2}}{\left|\omega^{2}\right|},
$$

and

$$
\Delta^{h}(K):=\min _{k \leq K-1}\left|\lambda_{k+1}^{h}-\lambda_{k}^{h}\right| .
$$

We are mainly interested in the values of $I_{\bullet}^{h}(N)$ (resp. $I_{\bullet}^{h}\left(k_{\text {max, }, ~}^{h}\right)$ ) in order to illustrate whether the corresponding quantities are bounded from below or not for the whole discrete spectrum (resp. for a constant portion of the spectrum characterized by $k_{\max , \varepsilon}^{h}$, see (29)) when the mesh size tends to 0 . In the tables below, each righthand side sub-column (with a white background) corresponds to the whole spectrum estimate (i.e. for $K=N$ ) whereas the left-hand side sub-column (with a gray background) corresponds to the partial spectrum estimate (i.e. for $K=k_{\max , \varepsilon}^{h}$ ). We have chosen $\varepsilon=0.05$, except for some cases for which it is explicitely mentioned.

Case 1. We consider $\gamma(x)=2+\cos (\pi x)^{3}, q(x)=0$ and a family of uniform meshes. This case corresponds to the framework (S2) and to Theorem 3.2.

\begin{tabular}{|c|c|c|c|c|c|c|c|c|c|c|c|}
\hline$N$ & $k_{\max , \varepsilon}^{h}$ & \multicolumn{2}{|c|}{$I_{l}^{h}(\cdot)$} & \multicolumn{2}{c|}{$I_{r}^{h}(\cdot)$} & \multicolumn{2}{c|}{$I_{1}^{h}(\cdot)$} & \multicolumn{2}{c|}{$I_{2}^{h}(\cdot)$} & \multicolumn{2}{c|}{$\Delta^{h}(\cdot)$} \\
\hline & & $k_{\max , \varepsilon}^{h}$ & $N$ & $k_{\max , \varepsilon}^{h}$ & $N$ & $k_{\max , \varepsilon}^{h}$ & $N$ & $k_{\max , \varepsilon}^{h}$ & $N$ & $k_{\max , \varepsilon}^{h}$ & $N$ \\
50 & 26 & 2.99 & 2.99 & 7.07 & $1.01_{-23}$ & 0.29 & 0.29 & 0.86 & $8.71_{-29}$ & 56.82 & 56.82 \\
100 & 52 & 2.99 & 2.99 & 7.08 & $2.46_{-51}$ & 0.28 & 0.28 & 0.85 & $1.26_{-59}$ & 56.89 & 56.89 \\
200 & 104 & 2.99 & 2.99 & 7.08 & $4.16_{-107}$ & 0.28 & 0.28 & 0.85 & $1.97_{-121}$ & 56.91 & 56.91 \\
300 & 156 & 2.99 & 2.99 & 7.08 & $4.22_{-163}$ & 0.28 & 0.28 & 0.84 & $2.70_{-183}$ & 56.91 & 56.91 \\
400 & 208 & 2.99 & 2.99 & 7.08 & $3.47_{-219}$ & 0.28 & 0.28 & 0.84 & $3.50_{-245}$ & 56.91 & 56.91 \\
\hline
\end{tabular}

Table 1: Case 1 - behavior as $h \rightarrow 0$

We observe in Table 1 that, in accordance with our theoretical results, all the partial spectrum quantities computed with $K=k_{\max , \varepsilon}^{h}$ (which is almost equal to $N / 2$ here) are bounded from below.

Interestingly enough, we observe that $I_{l}^{h}(N)$ and $I_{1}^{h}(N)$ are also uniformly bounded from below but it is not the case for $I_{r}^{h}(N)$ and $I_{2}^{h}(N)$. This discrepancy seems to come from the fact that the diffusion coefficient $\gamma$ is decreasing and maximal exactly on the left boundary of the domain. For those latter quantities, the only theoretical result we have is the one of Theorem 3.1. We can check numerically that the exponential bound given in this result seems to be sharp. Indeed we have observed that, 
for all the considered values of $N$, we have

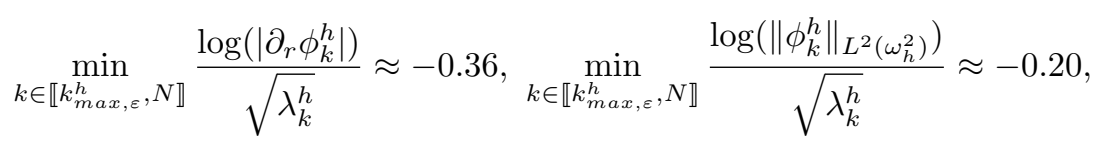

so that the quantities of interest actually behaves, in the upper part of the discrete spectrum, like $e^{-C \sqrt{\lambda_{k}^{h}}}$ for some $C$ independent of $h$.

Moreover, we observe in Figure $2 \mathrm{~b}$ that the actual value of $k_{\max , \varepsilon}^{h}$ is sharp and that $\partial_{r} \phi_{k}^{h}$ becomes exponentially small as soon as $k \gtrsim k_{\max , 0}^{h}$. However, in Figure 2c the exponential behavior of $\left\|\phi_{k}^{h}\right\|_{L^{2}\left(\omega_{h}^{2}\right)}$ seems to appear for higher values of $k$. This can be explained (and actually it can be deduced by a careful observation of our proofs) by the fact that, due to the monotonicity of $\gamma$, the minimal value of $\gamma$ that has to be taken into account in the evaluation of $k_{\max , \varepsilon}^{h}$ is not its infimum on $\Omega$ but rather its infimum on $\Omega \backslash \omega^{2}$. The correct threshold in that case is thus the one defined by

$$
\widetilde{k}_{\max , \varepsilon}^{h}:=\max \left\{k \in \llbracket 1, N \rrbracket ; \lambda_{k}^{h}<\frac{4}{h^{2}}\left(\inf _{\Omega \backslash \omega^{2}} \gamma\right)(1-\varepsilon)\right\} .
$$

We represent the value of $\widetilde{k}_{\max , 0}^{h}$ in Figure $2 \mathrm{a}$ and 2c.

Last but not least, we observe on this particular example that the uniform gap condition seems to be satisfied. In particular, by the methods given in Section 5, we can then conjecture that the associated parabolic equations or systems are indeed uniformly null-controllable by either distributed or boundary control.

Case 2. We consider now a case which is very much similar to the previous one by setting $\gamma(x)=2-\cos (2 \pi x)^{2}, q(x)=0$ and again a family of uniform meshes. The results are gathered in Table 2 . The main difference with the previous case lie in the fact that $\gamma$ is no more monotonic and is actually symmetric with respect to the mid-point $x=1 / 2$ (see Fig. 3a). It appears, in this configuration, that the uniform gap property does not hold anymore and that neither $I_{1}^{h}(N)$ nor $I_{2}^{h}(N)$ are uniformly bounded from below. We illustrate further those behaviors by plotting the difference between two successive eigenvalues in Fig. 3b as well as the last two eigenfunctions in Fig. 3c. We observe that those two eigenfunctions are essentially supported in one half of the domain (which explains why their $L^{2}$ norm on $\omega^{1}$ or $\omega^{2}$ can be exponentially small) and that they are almost symmetric to each other (which explains that the corresponding eigenvalues are very close).

\begin{tabular}{|c|c|c|c|c|c|c|c|c|c|c|c|}
\hline$N$ & $k_{\text {max }, \varepsilon}^{h}$ & \multicolumn{2}{|c|}{$I_{l}^{h}(\cdot)$} & \multicolumn{2}{c|}{$I_{r}^{h}(\cdot)$} & \multicolumn{2}{c|}{$I_{1}^{h}(\cdot)$} & \multicolumn{2}{|c|}{$I_{2}^{h}(\cdot)$} & \multicolumn{2}{|c|}{$\Delta^{h}(\cdot)$} \\
\hline & & $k_{\max , \varepsilon}^{h}$ & $N$ & $k_{\text {max }, \varepsilon}^{h}$ & $N$ & $k_{\text {max }, \varepsilon}^{h}$ & $N$ & $k_{\max , \varepsilon}^{h}$ & $N$ & $k_{\max , \varepsilon}^{h}$ & $N$ \\
50 & 32 & 6.39 & $1.74_{-3}$ & 6.39 & $1.74_{-3}$ & 0.56 & 0.56 & 0.6 & 0.6 & 33.53 & $4.51_{-8}$ \\
100 & 64 & 6.41 & $6.85_{-15}$ & 6.41 & $7.18_{-30}$ & 0.59 & $1.75_{-30}$ & 0.59 & $2.83_{-42}$ & 33.58 & $2.91_{-11}$ \\
200 & 126 & 6.42 & $3.02_{-63}$ & 6.42 & $3.80_{-14}$ & 0.58 & $8.90_{-87}$ & 0.58 & $2.81_{-30}$ & 33.59 & $2.91_{-11}$ \\
300 & 187 & 6.42 & $8.41_{-15}$ & 6.42 & $9.47_{-97}$ & 0.61 & $4.41_{-30}$ & 0.58 & $1.60_{-131}$ & 33.59 & $1.16_{-10}$ \\
400 & 250 & 6.42 & $2.50_{-130}$ & 6.42 & $5.30_{-15}$ & 0.58 & $7.38_{-176}$ & 0.58 & $6.02_{-30}$ & 33.59 & $6.98_{-10}$ \\
\hline
\end{tabular}

Table 2: Case 2 - behavior as $h \rightarrow 0$

Case 3. In this case we consider a constant diffusion coefficient $\gamma=1$ but a variable potential $q(x)=50 \cos \left(\pi x^{2}\right)$. We propose two subcases: in Subcase 3.1 we 


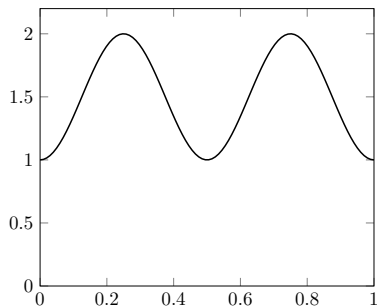

(a) The diffusion coefficient $\gamma$

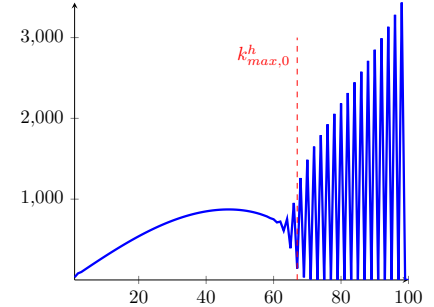

(b) $k \mapsto\left|\lambda_{k+1}^{h}-\lambda_{k}^{h}\right|$

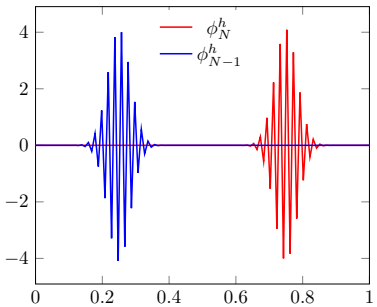

(c) The last two eigenfunctions

Figure 3: Case $2-\mathrm{N}=100$

still use uniform meshes families whereas in Subcase 3.2 we use families of quasiuniform meshes obtained by gluing a uniform mesh of $(0,1 / 2)$ with cells of size $h$ and a uniform mesh of $(1 / 2,1)$ made of cells of size $h / 2$; the value of $h$ is chosen so as we finally obtain the expected total number $N$ of cells. For this test case, we have chosen $\varepsilon=0.1$.

\begin{tabular}{|c|c|c|c|c|c|c|c|c|c|c|c|}
\hline$N$ & $k_{\text {max }, \varepsilon}^{h}$ & \multicolumn{2}{|c|}{$I_{l}^{h}(\cdot)$} & \multicolumn{2}{c|}{$I_{r}^{h}(\cdot)$} & \multicolumn{2}{c|}{$I_{1}^{h}(\cdot)$} & \multicolumn{2}{c|}{$I_{2}^{h}(\cdot)$} & \multicolumn{2}{c|}{$\Delta^{h}(\cdot)$} \\
\hline \multirow{5}{*}{50} & & $k_{\text {max }, \varepsilon}^{h}$ & $N$ & $k_{\text {max }, \varepsilon}^{h}$ & $N$ & $k_{\text {max }, \varepsilon}^{h}$ & $N$ & $k_{\text {max }, \varepsilon}^{h}$ & $N$ & $k_{\text {max }, \varepsilon}^{h}$ & $N$ \\
100 & 1.12 & 1.12 & 2.92 & 1.7 & $9.97_{-2}$ & $9.97_{-2}$ & 0.31 & $8.77_{-2}$ & 44.07 & 21.54 \\
200 & 86 & 1.12 & 1.12 & 2.92 & 1.7 & $9.73_{-2}$ & $9.73_{-2}$ & 0.31 & $8.66_{-2}$ & 44.21 & 21.56 \\
300 & 172 & 1.12 & 1.12 & 2.92 & 1.7 & $9.60_{-2}$ & $9.60_{-2}$ & 0.3 & $8.61_{-2}$ & 44.25 & 21.57 \\
400 & 257 & 1.12 & 1.12 & 2.92 & 1.7 & $9.55_{-2}$ & $9.55_{-2}$ & 0.3 & $8.59_{-2}$ & 44.26 & 21.57 \\
& 343 & 1.12 & 1.12 & 2.92 & 1.7 & $9.53_{-2}$ & $9.53_{-2}$ & 0.3 & $8.58_{-2}$ & 44.26 & 21.57 \\
\hline
\end{tabular}

Table 3: Subcase 3.1 - behavior as $h \rightarrow 0$

\begin{tabular}{|c|c|c|c|c|c|c|c|c|c|c|c|}
\hline$N$ & $k_{\max , \varepsilon}^{h}$ & \multicolumn{2}{|c|}{$I_{l}^{h}(\cdot)$} & \multicolumn{2}{c|}{$I_{r}^{h}(\cdot)$} & \multicolumn{2}{c|}{$I_{1}^{h}(\cdot)$} & \multicolumn{2}{c|}{$I_{2}^{h}(\cdot)$} & \multicolumn{2}{c|}{$\Delta^{h}(\cdot)$} \\
\hline \multirow{5}{*}{50} & & $k_{\max , \varepsilon}^{h}$ & $N$ & $k_{\max , \varepsilon}^{h}$ & $N$ & $k_{\max , \varepsilon}^{h}$ & $N$ & $k_{\max , \varepsilon}^{h}$ & $N$ & $k_{\max , \varepsilon}^{h}$ & $N$ \\
100 & 1.12 & $9.49_{-17}$ & 2.91 & 2.91 & 0.11 & $1.65_{-18}$ & 0.25 & $4.11_{-2}$ & 43.9 & 43.9 \\
200 & 50 & 1.12 & $9.55_{-16}$ & 2.92 & 2.92 & 0.1 & $1.74_{-30}$ & 0.13 & $1.04_{-2}$ & 44.17 & 44.17 \\
300 & 144 & 1.12 & $2.91_{-15}$ & 2.92 & 2.92 & $9.95_{-2}$ & $6.68_{-30}$ & 0.28 & $8.09_{-4}$ & 44.24 & 44.24 \\
400 & 1.12 & $1.24_{-14}$ & 2.92 & 2.92 & $9.71_{-2}$ & $1.32_{-29}$ & 0.25 & $3.35_{-4}$ & 44.25 & 44.25 \\
& 190 & 1.12 & $4.26_{-15}$ & 2.92 & 2.92 & $9.67_{-2}$ & $1.93_{-29}$ & 0.24 & $8.11_{-4}$ & 44.26 & 44.26 \\
\hline
\end{tabular}

Table 4: Subcase 3.2 - behavior as $h \rightarrow 0$

As predicted by Theorem 3.3 and Corollary 3.1, we observe in Table 3 that, in the subcase 3.1 all the quantities of interest are uniformly bounded from below. However, when considering a non uniform mesh we can see in Table 4 that the quantities $I_{l}^{h}(N)$ and $I_{1}^{h}(N)$ seem to be very small, but actually not exponentially small with respect to $\sqrt{\lambda}$. It is interesting to observe that the right derivative term $I_{r}^{h}(N)$ is still uniformly bounded from below as well as $I_{2}^{h}(N)$. This is an illustration of the fact that the bounds from below are more likely to be uniform when it concerns the finest part of 
the mesh than the coarsest part.

Case 4. We conclude this series of numerical illstrations by considering the case of a piecewise constant diffusion coefficient $\gamma=1_{] 0,0.4[}+2 \times 1_{] 0.4,1}$, with $q=0$ and a family of uniform meshes. This case does not directly enter our analysis but our arguments can be adapted and we can prove in that case (see Section 6, remark 2, for more details) that each quantity $I_{\bullet}^{h}(N)$ is

- either exponentially small if it concerns a part of the domain where $\gamma$ takes its lowest value (in the present example: the left normal derivative and the $L^{2}$ norm on $\left.\omega^{1}\right)$

- or uniformly bounded from below if it concerns a part of the domain where $\gamma$ takes its highest value (here : the right normal derivative and the $L^{2}$ norm on $\left.\omega^{2}\right)$.

\begin{tabular}{|c|c|c|c|c|c|c|c|c|c|c|c|c|}
\hline$N$ & $k_{\max , \varepsilon}^{h}$ & \multicolumn{2}{|c|}{$I_{l}^{h}(\cdot)$} & \multicolumn{2}{c|}{$I_{r}^{h}(\cdot)$} & \multicolumn{2}{c|}{$I_{1}^{h}(\cdot)$} & \multicolumn{2}{c|}{$I_{2}^{h}(\cdot)$} & \multicolumn{2}{c|}{$\Delta^{h}(\cdot)$} \\
\hline & & $k_{\max , \varepsilon}^{h}$ & $N$ & $k_{\max , \varepsilon}^{h}$ & $N$ & $k_{\max , \varepsilon}^{h}$ & $N$ & $k_{\max , \varepsilon}^{h}$ & $N$ & $k_{\max , \varepsilon}^{h}$ & $N$ \\
50 & 35 & 4.41 & $1.89_{-14}$ & 3.79 & 3.79 & 0.55 & $2.43_{-11}$ & 0.12 & 0.12 & 44.89 & 44.89 \\
100 & 68 & 5.37 & $9.21_{-30}$ & 3.79 & 3.79 & 0.68 & $7.18_{-20}$ & $5.87_{-2}$ & $5.87_{-2}$ & 44.62 & 44.62 \\
200 & 131 & 5.37 & $2.19_{-60}$ & 3.79 & 3.79 & 0.67 & $4.53_{-36}$ & $8.86_{-2}$ & $2.79_{-2}$ & 44.47 & 44.47 \\
300 & 194 & 5.37 & $5.22_{-91}$ & 3.79 & 3.79 & 0.67 & $6.63_{-52}$ & $9.81_{-2}$ & $1.88_{-2}$ & 44.42 & 44.42 \\
400 & 257 & 5.37 & $1.25_{-121}$ & 3.79 & 3.79 & 0.67 & $1.37_{-67}$ & 0.1 & $1.42_{-2}$ & 44.4 & 44.4 \\
\hline
\end{tabular}

Table 5: Case 4 - behavior as $h \rightarrow 0$

5. Applications in control theory. The moments method has been successfully used to prove null-controllability of parabolic equations and systems, in particular with boundary controls, see for instance $[13,3]$. In section 5.1 we present this method on the heat equation, then on cascade systems. Eventually, in Sections 5.2 and 5.3, we show how to adapt this strategy to the discrete setting.

5.1. Null-controllability via the moments method in the continuous setting. Let us fix $y^{0} \in H^{-1}(\Omega)$ and consider the following control problem

$$
\left\{\begin{array}{l}
\partial_{t} y(t, x)+\mathcal{A} y(t, x)=1_{\omega}(x) V_{d}(t, x), \text { in }(0, T) \times \Omega \\
y(t, 0)=0, y(t, 1)=V_{b}(t) \text { in }(0, T) \\
y(0, x)=y^{0}(x) \text { in } \Omega,
\end{array}\right.
$$

with $V_{d} \in L^{2}((0, T) \times \Omega), V_{b} \in L^{2}(0, T)$. Of course, all the results remain unchanged if one controls (48) at the left boundary instead of the right boundary.

For a given pair of controls $V_{b} \in L^{2}(0, T)$ and $V_{d} \in L^{2}((0, T) \times \Omega)$, we say that a function $y \in C^{0}\left([0, T], H^{-1}(\Omega)\right)$ is a solution of (48) if and only if for any $k \geq 1$ and any $t \in[0, T]$, we have

$$
\begin{aligned}
& \left\langle y(t), \phi_{k}\right\rangle_{H^{-1} \times H_{0}^{1}}-\left\langle y^{0}, e^{-\lambda_{k} t} \phi_{k}\right\rangle_{H^{-1} \times H_{0}^{1}} \\
& \quad=\int_{0}^{t} \int_{\Omega} 1_{\omega}(x) V_{d}(s, x) e^{-\lambda_{k}(t-s)} \phi_{k}(x) d x d s-\int_{0}^{t} \gamma(1) V_{b}(s) e^{-\lambda_{k}(t-s)} \partial_{x} \phi_{k}(1) d s,
\end{aligned}
$$

where $\left(\lambda_{k}, \phi_{k}\right)$ are the eigenelements of the self-adjoint operator $\mathcal{A}$ as defined in the introduction. 
It is proved in [11] that for any $V_{b}$ and $V_{d}$, such a solution exists and is unique. Since we are interested in the controllability of such a system at time $T$, we will often make use of the above formula specialized at time $t=T$

$$
\begin{aligned}
\left\langle y(T), \phi_{k}\right\rangle_{H^{-1}} \times H_{0}^{1} & -\left\langle y^{0}, e^{-\lambda_{k} T} \phi_{k}\right\rangle_{H^{-1} \times H_{0}^{1}} \\
& =\int_{0}^{T} \int_{\Omega} 1_{\omega}(x) V_{d}(t, x) z_{k}(t, x) d x d t-\int_{0}^{T} \gamma(1) V_{b}(t) \partial_{x} z_{k}(t, 1) d t,
\end{aligned}
$$

where we have defined $z_{k}$ by $z_{k}:(t, x) \mapsto e^{-\lambda_{k}(T-t)} \phi_{k}(x)$ which is nothing but the solution of the adjoint problem with final data $\phi_{k}$

$$
\left\{\begin{array}{l}
-\partial_{t} z_{k}(t, x)+\mathcal{A} z_{k}(t, x)=0 \text { in }(0, T) \times \Omega, \\
z_{k}(t, 0)=z_{k}(t, 1)=0 \text { in }(0, T), \\
z_{k}(T, x)=\phi_{k}(x) \text { in } \Omega .
\end{array}\right.
$$

- Consider first the right-boundary null-control problem : we set $V_{d}=0$, and we look for a boundary control $V_{b} \in L^{2}(0, T)$ such that the corresponding solution $y$ of (48) satisfies $y(T)=0$.

We write the decomposition of $y^{0}$ in the basis $\left(\phi_{k}\right)_{k \geq 1}$ as follows $y^{0}=$ $\sum_{k \geq 1} y_{k}^{0} \phi_{k}$ and from (49) we see that the problem amounts to finding $V_{b}$ such that

$$
y_{k}^{0} e^{-\lambda_{k} T}=\gamma(1) \partial_{r} \phi_{k}\left(\int_{0}^{T} V_{b}(t) e^{-\lambda_{k}(T-t)} d t\right), \forall k \geq 1 .
$$

The set of equations (51) indexed by $k$ is called a moment problem. The moments method consists in solving (51) using a biorthogonal family of the real exponentials $\left(e^{-\lambda_{k}(T-t)}\right)_{k \geq 1}$.

Let $\Lambda=\left(\lambda_{k}\right)_{k \geq 1}$ be a sequence of positive numbers. A biorthogonal family of $\left(e^{-\lambda_{k}(T-t)}\right)_{k \geq 1}$ is a set of functions in $L^{2}(0, T)$ denoted by $\left(q_{l}^{\Lambda}\right)_{l \geq 1}$ verifying

$$
\int_{0}^{T} e^{-\lambda_{k}(T-t)} q_{l}^{\Lambda}(t) d t=\delta_{l, k}, \quad \forall l, k \geq 1 .
$$

We can now solve, at least formally, the moment problem (51) and give a possible expression of $V_{b}$

$$
V_{b}(t):=\sum_{k \geq 1} \frac{y_{k}^{0} e^{-\lambda_{k} T}}{\gamma(1) \partial_{r} \phi_{k}} q_{k}^{\Lambda}(t)
$$

- Now, we study the distributed control problem: we set $V_{b}=0$ and we look for a control $V_{d}$ such that the corresponding solution $y$ of (48) satisfies $y(T)=0$. Using again (49), this amounts to finding $V_{d}$ satisfying the following family of equalities

$$
-y_{k}^{0} e^{-\lambda_{k} T}=\int_{0}^{T} \int_{\omega} V_{d}(t, x) e^{-\lambda_{k}(T-t)} \phi_{k}(x) d x d t, \quad \forall k \geq 1 .
$$

Inspired by the boundary control case, we look for a suitable $V_{d}$ in the following form

$$
V_{d}(t, x)=\sum_{k \geq 1} \alpha_{k} q_{k}^{\Lambda}(t) \phi_{k}(x),
$$


where $\left(\alpha_{k}\right)_{k \geq 1}$ is a sequence of real numbers to be determined.

Injecting (55) in (54) and using (52) we finally get the following formula for the unknown coefficients

$$
\alpha_{k}=-\frac{y_{k}^{0} e^{-\lambda_{k} T}}{\int_{\omega} \phi_{k}^{2}(x) d x} .
$$

Formally, the control problem is thus solved by defining

$$
V_{d}(t, x):=\sum_{k \geq 1}\left(-\frac{y_{k}^{0} e^{-\lambda_{k} T}}{\left\|\phi_{k}\right\|_{L^{2}(\omega)}^{2}}\right) q_{k}^{\Lambda}(t) \phi_{k}(x) .
$$

REMARK 5.1. This strategy is not classic. In many papers (see [3], [4], [13]) when $\mathcal{A}$ is the Laplace operator, the authors rather look for a distributed control of the form $V_{d}(t, x)=u(t) f(x)$, where $f$ is a well chosen profile function, supported in $\omega$, and satisfying some lower bounds for its generalized Fourier coefficients $\left|\int_{\Omega} f \phi_{k} d x\right|$. However, it is not straightforward to find such a $f$ when $\mathcal{A}$ is a more general elliptic operator as in (1), since we dot not have analytic formulas for the $\phi_{k}$ (and, a fortiori, finding the equivalent of $f$ in the discrete setting seems to be even more complicated).

Thus, the ansatz $V_{d}(t, x)=\sum_{k \geq 1} \alpha_{k} q_{k}^{\Lambda}(t) \phi_{k}(x)$ we choose here is somehow more convenient since it does not require to find such a function $f$. Notice that the family $(t, x) \mapsto q_{k}^{\Lambda}(t) \phi_{k}(x) /\left\|\phi_{k}\right\|_{L^{2}(\omega)}^{2}$ can be seen as a space-time biorthogonal family of $(t, x) \mapsto e^{-(T-t) \lambda_{k}} \phi_{k}(x)$ in $L^{2}((0, T) \times \omega)$.

This form of distributed control has been used in [16] to prove exact controllability of the 1d-wave equation.

To sum up, in order to justify the previous application of the moment method, we must check that

1. Such a biorthogonal family $\left(q_{k}^{\Lambda}\right)_{k}>1$ exists.

2. The formal series (53) (resp. (56)) that defines $V_{b}$ (resp. $V_{d}$ ) converges. To this end, we need

(a) to estimate the $L^{2}(0, T)$-norms of $q_{k}^{\Lambda}$, for all $k \geq 1$,

(b) to give lower bounds on $\left\|\phi_{k}\right\|_{L^{2}(\omega)}$ and $\left|\partial_{r} \phi_{k}\right|$, that appear at the denominator in those formulas.

The lower bounds in the point $2 \mathrm{~b}$ were stated in the assertions 2 and 3 of Theorem 1.1. We shall now tackle points 1 and $2 \mathrm{a}$ at the same time.

Problems of existence and bounds on the biorthogonal family have been studied in [13] and [2] and we recall below some useful results.

First, we need to extend the definition of a biorthogonal family given above, for the purpose of controlling systems of coupled parabolic equations.

Definition 5.1. Let $\Sigma:=\left(\sigma_{k}\right)_{k \geq 1}$ be a sequence of positive real numbers. Let $T>0$ and $m \in \mathbb{N}$. A biorthogonal family of $\left((t-T)^{i} e^{-\sigma_{k}(T-t)}\right)_{i \in \llbracket 0, m \rrbracket}^{k \geq 1}$ is a set of functions in $L^{2}(0, T)$ denoted by $\left(q_{j, l}^{\Sigma}\right)_{j \in \llbracket 0, m \rrbracket}^{l \geq 1}$ satisfying

$$
\int_{0}^{T}(t-T)^{i} \exp \left(-\sigma_{k}(T-t)\right) q_{j, l}^{\Sigma}(t) d t=\delta_{k, l} \delta_{i, j}, \quad \forall k, l \geq 1, \forall i, j \in \llbracket 0, m \rrbracket .
$$

Before stating an existence result and estimates for such biorthogonal families in Theorem 5.1, we need the following definition (see [13, Theorem 1.1]) 
Definition 5.2 (class of sequences $\mathcal{L}(\rho, \mathcal{N})$ ). Let $\rho>0$ and let $\mathcal{N}: \mathbb{R}^{+} \rightarrow \mathbb{N}$. We denote by $\mathcal{L}(\rho, \mathcal{N})$ the class of all sequences of positive numbers $\Sigma=\left(\sigma_{k}\right)_{k \geq 1}$ that satisfy the conditions:

$$
\begin{gathered}
\sigma_{k+1}-\sigma_{k} \geq \rho, \quad \forall k \geq 1, \\
\sum_{k=\mathcal{N}(\xi)}^{\infty} \frac{1}{\sigma_{k}} \leq \xi, \quad \forall \xi>0 .
\end{gathered}
$$

TheOREM 5.1. Let $T>0$ and $m \in \mathbb{N}$. Let $\rho>0$ and $\mathcal{N}: \mathbb{R}_{+} \rightarrow \mathbb{N}$.

For any $\tau>0$, there exists $K(\tau, T, \rho, \mathcal{N}, m)>0$ such that for any sequence $\Sigma:=\left(\sigma_{k}\right)_{k \geq 1}$ in the class $\mathcal{L}(\rho, \mathcal{N})$, there exists a biorthogonal family $\left(q_{j, l}^{\Sigma}\right)_{j \in \llbracket 10, m \rrbracket}^{l \geq 1}$ in $L^{2}(0, T)$ for $\left((t-T)^{i} e^{-\sigma_{k}(T-t)}\right)_{i \in \llbracket 0, m \rrbracket}^{k \geq 1}$ such that

$$
\left\|q_{j, l}^{\Sigma}\right\|_{L^{2}(0, T)} \leq K e^{\tau \sigma_{l}}, \quad \forall l \geq 1, \forall j \in \llbracket 0, m \rrbracket .
$$

REMARK 5.2. We emphasize that the upper bound $K e^{\tau \sigma_{l}}$ does not depend on the choice of the particular sequence $\Sigma$ in the class $\mathcal{L}(\rho, \mathcal{N})$. Thus, if there exist $\rho$ and $\mathcal{N}$ such that $\forall h>0, \Lambda^{h} \in \mathcal{L}(\rho, \mathcal{N})$, then the sequences $\left\|q_{j, l}^{\Lambda^{h}}\right\|_{L^{2}(0, T)}$ are uniformly bounded with respect to parameter $h$. This point is essential to get uniform bounds with respect to $h$ on boundary and distributed controls $V_{b}^{h}$ and $V_{d}^{h}$.

For $m=0$, this result is proved in [13, Theorem 1.1] and for $m \geq 1$ it is proved in [2, Theorem 1.2]. In this last reference, the dependence of the bounds with respect to the sequence $\Sigma$ is not precised but a careful inspection of the proof shows that the constants are actually uniform in the class $\mathcal{L}(\rho, \mathcal{N})$ as in [13].

We can now completely justify the moments method applied to system (48) that we described formally above. For the sake of completeness, we state the following theorem in the more general case of a cascade system of $d \geq 1$ parabolic equations. We introduce a control vector $B \in \mathbb{R}^{d}$ and a coupling $d \times d$ matrix $C$ defined as follows

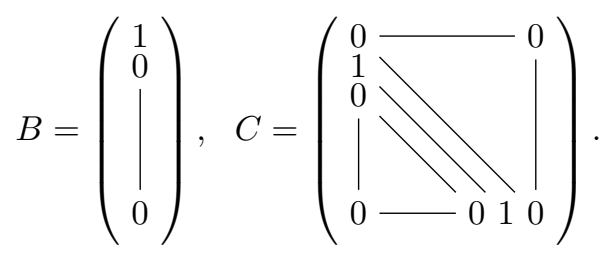

Let $\omega$ be a non empty open subset of $\Omega$ and $T>0$. For any $Y^{0} \in\left(H^{-1}(\Omega)\right)^{d}$, $V_{b} \in L^{2}(0, T), V_{d} \in L^{2}((0, T) \times \Omega)$ the problem

$$
\left\{\begin{array}{l}
\partial_{t} Y+\mathcal{A} Y+C Y=1_{\omega} B V_{d}(t, x) \text { in }(0, T) \times \Omega \\
Y(t, 0)=0, \quad Y(t, 1)=B V_{b}(t) \text { in }(0, T) \\
Y(0, x)=Y^{0}(x) \text { in } \Omega
\end{array}\right.
$$

has a unique solution in $\mathcal{C}^{0}\left([0, T],\left(H^{-1}(\Omega)\right)^{d}\right)$. Observe that $Y$ has $d$ components (and $\mathcal{A}$ acts component-by-component on $Y$ ) but the controls $V_{b}$ and $V_{d}$ are scalar.

Theorem 5.2. Assume that $\left(\mathrm{H}_{0}\right)$ holds. For any $Y^{0} \in\left(H^{-1}(\Omega)\right)^{d}$, System (57) is null controllable at time $T$ with either a distributed control $V_{d} \in L^{2}((0, T) \times \Omega)$ (in this case, we set $V_{b}=0$ ) or a boundary control $V_{b} \in L^{2}(0, T)$ (in this case, we set $\left.V_{d}=0\right)$. 
REMARK 5.3. Notice that when $d=1$, system (57) is nothing but system (48).

Notice also that, as usual in the study of controllability properties of systems with fewer controls than equations, not every equation is directly controlled. Indeed, the control directly intervenes only in the evolution equation for the first component of the solution. Thanks to the particular structure of the coupling matrix $C$, the second component is indirectly controlled by means of the first component itself and so on.

Proof. As an example, we start by considering the case $d=1$. The first assertion of Theorem 1.1 as well as (2) ensure that Theorem 5.1 applies with $m=0$ and $\Sigma=\Lambda$. Choosing for instance $\tau=T / 2$, and using the second and third assertions of Theorem 1.1 , one can easily check that the series (53) (resp. the series (56)) converges in $L^{2}(0, T)$ (resp. in $L^{2}((0, T) \times \Omega)$, which justifies the formal approach and proves the claim.

The same kind of arguments apply to the case $d>1$. First, note that the eigenvalues of the adjoint operator $\mathcal{L}:=\mathcal{A}+{ }^{t} C$ in $\left(L^{2}(\Omega)\right)^{d}$ are the $\left(\lambda_{k}\right)_{k \geq 1}$, as in the case $d=1$. They are geometrically simple and possess a $d \times d$ Jordan bloc. More precisely if we define, $\Phi_{k}^{r}:=\phi_{k} e_{r}$ for $r \in \llbracket 1, d \rrbracket$ where $\left(e_{1}, \ldots, e_{d}\right)$ is the canonical basis of $\mathbb{R}^{d}$ and $\Phi_{k}^{0}=0$, then we have

$$
\mathcal{L} \Phi_{k}^{r}=\mathcal{A} \Phi_{k}^{r}+{ }^{t} C \Phi_{k}^{r}=\lambda_{k} \Phi_{k}^{r}+\Phi_{k}^{r-1}, \quad \forall r \in \llbracket 1, d \rrbracket,
$$

so that $\Phi_{k}^{1}$ is an eigenfunction, and the $\Phi_{k}^{r}, r \geq 2$ are generalized eigenfunctions.

With those notations we can explicitely compute the semi-group associated with $\mathcal{L}$ for each initial data $\Phi_{k}^{r}$, as follows

$$
e^{-s \mathcal{L}} \Phi_{k}^{r}=\sum_{l=0}^{r-1} \frac{(-s)^{l}}{l !} e^{-\lambda_{k} s} \Phi_{k}^{r-l}, \quad \forall s \geq 0 .
$$

- The right-boundary null-control problem consists in finding a control $V_{b} \in$ $L^{2}(0, T)$ such that $\forall k \geq 1, \forall r \in \llbracket 1, d \rrbracket$,

$$
\left\langle Y^{0}, e^{-T \mathcal{L}} \Phi_{k}^{r}\right\rangle_{H^{-1} \times H_{0}^{1}}=\sum_{l=0}^{r-1}\left(B, \partial_{x} \Phi_{k}^{r-l}(1)\right) \gamma(1) \int_{0}^{T} V_{b}(t) \frac{(t-T)^{l}}{l !} e^{-\lambda_{k}(T-t)} d t,
$$

where $(\cdot, \cdot)$ denotes the canonical inner product of $\mathbb{R}^{N}$. By definition of $B$ and $\Phi_{k}^{r-1}$, we see that $\left(B, \partial_{x} \Phi_{k}^{r-l}(1)\right)=\delta_{l, r-1} \partial_{x} \phi_{k}(1)$. We are thus led to solve the following moments problem: find $V_{b} \in L^{2}(0, T)$ such that for any $k \geq 1$ and $r \in \llbracket 1, d \rrbracket$

$$
\left\langle Y^{0}, e^{-T \mathcal{L}} \Phi_{k}^{r}\right\rangle_{H^{-1} \times H_{0}^{1}}=\frac{\gamma(1) \partial_{r} \phi_{k}}{(r-1) !} \int_{0}^{T} V_{b}(t)(t-T)^{r-1} e^{-\lambda_{k}(T-t)} d t .
$$

The gap estimate in Theorem 1.1 (first assertion) and the inequality (2) ensure that we can apply Theorem 5.1 with $m=d-1$ and $\tau=T / 2$ (for instance) and obtain a biorthogonal family whose norms are bounded by $e^{\lambda_{k} T / 2}$. Then, we observe that

$$
\left\|e^{-T \mathcal{L}} \Phi_{k}^{r}\right\|_{H_{0}^{1}} \leq e^{-\lambda_{k} T}\left(\sum_{l=0}^{r-1} \frac{T^{l}}{l !}\right)\left\|\phi_{k}\right\|_{H_{0}^{1}} \leq C e^{-\lambda_{k} T} \sqrt{\lambda_{k}} .
$$


Finally, with the uniform lower bound on $\partial_{r} \phi_{k}$ given in Theorem 1.1 we can conlude that the following definition of $V_{b}$

$$
V_{b}(t)=\sum_{k \geq 1} \sum_{r=1}^{d}(r-1) ! \frac{\left\langle-Y^{0}, e^{-T \mathcal{L}} \Phi_{k}^{r}\right\rangle_{H^{-1} \times H_{0}^{1}}}{\gamma(1) \partial_{r} \phi_{k}} q_{r-1, k}^{\Lambda}(t),
$$

is actually a series that converges in $L^{2}(0, T)$ and which is a solution of our boundary control problem.

- Considering now the distributed control problem, we look for a control $V_{d}$ of the following form

$$
V_{d}(t, x)=\sum_{k \geq 1} \sum_{r=1}^{d} \alpha_{k, r} q_{r-1, k}^{\Lambda}(t) \phi_{k}(x)
$$

with the coefficients $\alpha_{k, r}$ to be determined. By using formally this expression in the weak formulation of the problem, we obtain

$$
\alpha_{k, r}=(r-1) ! \frac{\left\langle-Y^{0}, e^{-T \mathcal{L}} \Phi_{k}^{r}\right\rangle_{H^{-1} \times H_{0}^{1}}}{\left\|\phi_{k}\right\|_{L^{2}(\omega)}^{2}}, \forall k \geq 1, \forall r \in \llbracket 1, d \rrbracket .
$$

Still using the bounds on the biorthogonal family $\left(q^{\Lambda}\right)_{r \in \llbracket 0, d-1 \rrbracket}^{k \geq 1}$, the bound on $\left\|e^{-T \mathcal{L}} \Phi_{k}^{r}\right\|_{H_{0}^{1}}$ and the uniform lower bound on $\left\|\phi_{k}\right\|_{L^{2}(\omega)}$ given in Theorem 1.1, we obtain that the series

$$
V_{d}(t, x)=\sum_{k \geq 1} \sum_{r=1}^{d}(r-1) ! \frac{\left\langle-Y^{0}, e^{\left.-T \mathcal{L}_{\Phi^{\prime}}^{r}\right\rangle_{H^{-1} \times H_{0}^{1}}}\right.}{\left\|\phi_{k}\right\|_{L^{2}(\omega)}^{2}} q_{r-1, k}^{\Lambda}(t) \phi_{k}(x)
$$

converges in $L^{2}((0, T) \times \Omega)$ and is solution to our distributed control problem.

$\square$

5.2. Null controllability in the discrete setting. We now want to apply this strategy to a discrete version of (57). For a given mesh, we consider the discrete control problem

$\left(S_{d}^{h}\right) \quad\left\{\begin{array}{l}\left(Y^{h}\right)^{\prime}(t)+\mathcal{A}^{h} Y^{h}(t)+\mathrm{C}^{h} Y^{h}(t)=\mathrm{B}^{h}\left(\mathcal{D}_{\omega}^{h} V_{\mathrm{d}}^{h}(t)+\mathcal{B}_{r}^{h} V_{\mathrm{b}}^{h}(t)\right), \text { for } 0<t \leq T, \\ Y^{h}(0)=Y^{0, h} \in\left(\mathbb{R}^{N}\right)^{d},\end{array}\right.$

where $\mathrm{B}^{h}$ is the $(N d) \times N$ matrix and $\mathrm{C}^{h}$ the $(N d) \times(N d)$ matrix given by

$$
\mathrm{B}^{h}=\left(\begin{array}{c}
\mathrm{I}^{h} \\
0^{h} \\
\mid \\
0^{h}
\end{array}\right), \quad \mathrm{C}^{h}=\left(\begin{array}{c}
0^{h}-0^{h} \\
\mathrm{I}^{h} \\
0^{h} \\
\mid \backslash \\
0^{h}-0^{h} \mathrm{I}^{h} 0^{h}
\end{array}\right),
$$

that approximate the control vector $B$ and the coupling matrix $C$. Here we still use the notation $\mathcal{A}^{h}$ for the component-by-component discrete elliptic operator. 
The solutions of this system satisfy the discrete analogue of (49) that is: $\forall k \in$ $\llbracket 1, N \rrbracket, \forall r \in \llbracket 1, d \rrbracket$,

$$
\begin{aligned}
\left\langle Y^{h}(T), \Phi_{k}^{r, h}\right\rangle_{L^{2}\left(\Omega_{h}\right)} & -\left\langle Y^{0, h}, e^{-T \mathcal{L}^{h}} \Phi_{k}^{r, h}\right\rangle_{L^{2}\left(\Omega_{h}\right)} \\
= & \int_{0}^{T}\left\langle\mathrm{~B}^{h} \mathcal{D}_{\omega}^{h} V_{d}^{h}(t), e^{-(T-t) \mathcal{L}^{h}} \Phi_{k}^{r, h}\right\rangle_{L^{2}\left(\Omega_{h}\right)} d t \\
& \quad-\int_{0}^{T} V_{b}^{h}(t) \gamma_{N+1 / 2} \partial_{r} \phi_{k}^{h} \frac{(t-T)^{r-1}}{(r-1) !} \exp \left(-\lambda_{k}^{h}(T-t)\right) d t
\end{aligned}
$$

where $\mathcal{D}_{\omega}^{h}$ is defined in (7). Here, $\Phi_{k}^{r, h}$ is a column vector of size $d N$ and $\left(\Phi_{k}^{r, h}\right)_{i}=$ $\left(\phi_{k}^{h}\right)_{i-(r-1) N}$ for $i \in \llbracket 1+(r-1) N, r N \rrbracket$ and 0 otherwise.

We have used here that the semi-group associated with the adjoint operator $\mathcal{L}^{h}:=$ $\mathcal{A}^{h}+{ }^{t} \mathrm{C}^{h}$ satisfies

$$
e^{-s \mathcal{L}^{h}} \Phi_{k}^{r, h}=\sum_{l=0}^{r-1} \frac{(-s)^{l}}{l !} e^{-\lambda_{k}^{h} s} \Phi_{k}^{r-l, h} .
$$

In the discrete setting, we not only want to control the discrete system for any mesh but also to have uniform bounds on them, with respect to $h$, in order to be able to conclude that, at least in a weak sense, the discrete controls will eventually converge towards a control of the continuous problem.

Therefore, in this setting, for a given family of initial data $\left(Y^{0, h}\right)_{h}$, the null-control problem consists in finding distributed controls $\left(V_{d}^{h}\right)_{h>0}$ (resp. boundary controls $\left.\left(V_{b}^{h}\right)_{h>0}\right)$ such that the corresponding solution $Y^{h}$ with $V_{b}^{h}=0\left(\right.$ resp. $\left.V_{d}^{h}=0\right)$ satisfies

$$
Y^{h}(T)=0, \forall h>0,
$$

and such that, for some $C>0$ depending only on the data $(\gamma, q, \omega, \beta$ and so on), $\left(V_{d}^{h}\right)_{h>0}$ and $\left(V_{b}^{h}\right)_{h>0}$ satisfy

$$
\left\|V_{d}^{h}\right\|_{L^{2}\left(\Omega_{h}^{T}\right)} \leq C\left\|Y^{0, h}\right\|_{L^{2}\left(\Omega_{h}\right)}, \text { and }\left\|V_{b}^{h}\right\|_{L^{2}(0, T)} \leq C\left\|Y^{0, h}\right\|_{L^{2}\left(\Omega_{h}\right)}, \quad \forall h>0 .
$$

In the discrete case, we will refer to uniform null controllability as the combination of condition (61) and (62). We start by considering the setting (S3), which is the simplest one.

TheOREM 5.3. Let $d \in \mathbb{N}^{*}$ and $T>0$. Consider a uniform mesh and suppose that $\gamma$ is a constant function, while $q$ is any continuous function. Then, the discrete cascade system $\left(S_{d}^{h}\right)$ is uniformly null controllable at time $T$ either with a distributed control, or with a boundary control.

Proof. Let us introduce the sequence

$$
\tilde{\Lambda}^{h}:=\left\{\begin{array}{l}
\lambda_{k}^{h} \text { for } k \in \llbracket 1, N \rrbracket \\
\lambda_{N}^{h}+4 \gamma k^{2} \text { for } k \geq N+1 .
\end{array}\right.
$$

Notice that $\left(\tilde{\Lambda}^{h}\right)_{k} \geq 4 \gamma k^{2}-\|q\|_{\infty}$ for any $k \geq 1$ (see Lemma 3.3 and Remark 1.3). First, we prove that there exists $\rho>0$ and $\mathcal{N}$, an integer-valued function, such that $\tilde{\Lambda}^{h} \in \mathcal{L}(\rho, \mathcal{N})$ for any $h>0$. Let $\mathcal{N}: \mathbb{R}_{+} \rightarrow \mathbb{N}$ be a function satisfying

$$
\sum_{k \geq \mathcal{N}(\eta)} \frac{1}{4 \gamma k^{2}-\|q\|_{\infty}} \leq \eta, \forall \eta>0
$$


According to Corollary 3.1 we know that there exists $\kappa>0$ such that, for any $h>0$, we have

$$
\lambda_{k+1}^{h}-\lambda_{k}^{h} \geq \kappa, \forall k \in \llbracket 1, N-1 \rrbracket .
$$

One can check that

$$
\tilde{\Lambda}^{h} \in \mathcal{L}(\min (\kappa, 4 \gamma), \mathcal{N}), \forall h>0 .
$$

Thus, we can apply Theorem 5.1 which states that given any $\tau \in(0, T)$, there exists a $K>0$, such that for and any $h>0$, there exists a biorthogonal family $\left(q_{r, k}^{\tilde{\Lambda}^{h}}\right)_{r \in \llbracket 0, d-1 \rrbracket}^{k \in \llbracket 1, N \rrbracket}$ satisfying

$$
\left\|q_{r, k}^{\tilde{\Lambda}^{h}}\right\|_{L^{2}(0, T)} \leq K e^{\tau \lambda_{k}^{h}}, \forall h>0, \forall k \in \llbracket 1, N \rrbracket, \forall r \in \llbracket 0, d-1 \rrbracket .
$$

It is fundamental to notice that the upper bound in (66) is valid for any $h>0$ even though the sequence of eigenvalues depends on $h$, thanks to (65), see also Remark 5.2.

From equation (60) and adapting the strategy of Section 5.1, we find the following expressions

$$
V_{b}^{h}(t)=\sum_{k=1}^{N} \sum_{r=1}^{d}(r-1) ! \frac{\left\langle Y^{0, h}, e^{-T \mathcal{L}^{h}} \Phi_{k}^{h, r}\right\rangle_{L^{2}\left(\Omega_{h}\right)}}{\gamma \partial_{r} \phi_{k}^{h}} q_{r-1, k}^{\tilde{\Lambda}^{h}}(t), \text { and } V_{d}^{h}(t)=0,
$$

for the boundary control case and

$$
V_{d}^{h}(t)=\sum_{k=1}^{N} \sum_{r=1}^{d}(r-1) ! \frac{-\left\langle Y^{0, h}, e^{-T \mathcal{L}^{h}} \Phi_{k}^{h, r}\right\rangle_{L^{2}\left(\Omega_{h}\right)}}{\left\|\phi_{k}^{h}\right\|_{L^{2}\left(\omega_{h}\right)}^{2}} q_{r-1, k}^{\tilde{\Lambda}^{h}}(t) \phi_{k}^{h}, \text { and } V_{b}^{h}(t)=0 .
$$

for the distributed control case.

We deal now with finite sums and there is no series convergence problem as soon as $\partial_{r} \phi_{k}^{h} \neq 0$ (this is alway true) and $\left\|\phi_{k}^{h}\right\|_{L^{2}\left(\omega_{h}\right)} \neq 0$ (which is true as soon as $\omega$ contains at least two different points of the mesh, see Remark 1.4).

However, it remains to check that condition (62) is satisfied. According to the lower bounds in Corollary 3.1 and to (66), we find that for any $h>0$, the distributed control in (68) satisfies

$$
\left\|V_{d}^{h}\right\|_{L^{2}\left(\Omega_{h}^{T}\right)} \leq C\left\|Y^{0, h}\right\|_{L^{2}\left(\Omega_{h}\right)} \sum_{k=1}^{N} \lambda_{k}^{h} e^{-(T-\tau) \lambda_{k}^{h}} \leq C\left\|Y^{0, h}\right\|_{L^{2}\left(\Omega_{h}\right)} \sum_{k \geq 1} k^{2} e^{-(T-\tau) 4 \gamma k^{2}},
$$

and thus,

$$
\left\|V_{d}^{h}\right\|_{L^{2}\left(\Omega_{h}^{T}\right)} \leq C\left\|Y^{0, h}\right\|_{L^{2}\left(\Omega_{h}\right)}
$$

The same computations hold for $\left(V_{b}^{h}\right)_{h>0}$ in (67).

REMARK 5.4. Notice that, for the problem of control under consideration, we did not need so much precision on the lower bounds of Corollary 3.1. Indeed, controls $\left(V_{b}^{h}\right)_{h>0}$ and $\left(V_{d}^{h}\right)_{h>0}$ satisfy condition (62) as soon as the sums (67) and (68) are bounded uniformly in $h$. Thus, for this purpose, results of Theorem 3.1 would have been enough. 
5.3. $\phi(h)$-null controllability. As already mentioned in the last paragraph of Section 1.3.1 and observed in numerical simulations of Section 4, the gap property (4) as well as the uniform lower bounds for $\left|\partial_{b} \phi_{k}^{h}\right|$ or $\left\|\phi_{k}^{h}\right\|_{L^{2}\left(\omega_{h}\right)}$ may not be satisfied when the diffusion coefficient is not constant or when the mesh is not uniform. Nevertheless, by Remark 1.4, we may still follow the proof of Theorem 5.3 exactly the same way and produce semi-discrete controls for which the associated solution satisfies $Y^{h}(T)=0$. Unfortunately, due to the lack of uniformity for the spectral properties mentionned above, we will not be able to produce a uniform bound for the control costs $\left\|V_{d}^{h}\right\|_{L^{2}\left(\Omega_{h}^{T}\right)}$ or $\left\|V_{b}^{h}\right\|_{L^{2}(0, T)}$. It follows that such semi-discrete null-controls may be unstable when $h \rightarrow 0$.

Therefore, in these latter cases, we will no longer look for controls that lead to (61) and instead, we consider an adapted weaker definition of null-controllability, namely, we now investigate the $\phi(h)$-null controllability problem (see Definition 1.1).

REMARK 5.5. Note that the property $\left\|Y^{h}(T)\right\|_{L^{2}\left(\Omega_{h}\right)}^{2} \leq C \phi(h)\left\|Y^{0, h}\right\|_{L^{2}\left(\Omega_{h}\right)}^{2}$ ensures that $Y^{h}(T) \rightarrow 0$ as $h \rightarrow 0$ if the family of discrete initial data are bounded. Thus, the $\phi(h)$-null controllability problem really aims at approaching null-controls in the limit $h \rightarrow 0$ and not approximate controls.

Let us first state a lemma on which the proofs of Theorem 5.4 and Theorem 5.5 both rely.

Lemma 5.1. Let $t_{0} \in(0, T), K \geq 1$, and $\beta>0$. There exists a $C\left(q, \gamma, t_{0}, \beta\right)>0$ such that

- for any mesh satisfying $\Theta_{h} \leq \beta$ and $N>K$

- for any $V_{d}^{h} \in L^{2}\left(0, T, \mathbb{R}^{N}\right)$ and $V_{b}^{h} \in L^{2}(0, T)$ that vanish on $\left(t_{0}, T\right)$ for which the associated solution $Y^{h}$ of $\left(S_{d}^{h}\right)$ satisfies

$$
\left\langle\Phi_{k}^{r, h}, Y^{h}\left(t_{0}\right)\right\rangle_{L^{2}\left(\Omega_{h}\right)}=0, \forall k \in \llbracket 1, K \rrbracket, \forall r \in \llbracket 1, d \rrbracket,
$$

we have

$$
\left\|Y^{h}(T)\right\|_{L^{2}\left(\Omega_{h}\right)} \leq C e^{-\left(T-t_{0}\right) \lambda_{K+1}^{h}}\left(\left\|Y^{0, h}\right\|_{L^{2}\left(\Omega_{h}\right)}+\frac{1}{h^{3 / 2}}\left\|V_{b}^{h}\right\|_{L^{2}(0, T)}+\left\|V_{d}^{h}\right\|_{L^{2}\left(\Omega_{h}^{T}\right)}\right) .
$$

Proof. Since the family $\left(\Phi_{k}^{r, h}\right)_{r \in \llbracket 1, d \rrbracket}^{k \in \llbracket 1, N \rrbracket}$, is an orthonormal basis of $\mathbb{R}^{N d}$, we have

$$
\left\|Y^{h}\left(t_{0}\right)\right\|_{L^{2}\left(\Omega_{h}\right)}^{2}=\sum_{k=1}^{N} \sum_{r=1}^{d}\left\langle Y^{h}\left(t_{0}\right), \Phi_{k}^{r, h}\right\rangle_{L^{2}\left(\Omega_{h}\right)}^{2} .
$$

Now, using (60) with $T$ replaced by $t_{0}$ we find that

$$
\begin{aligned}
& \left\|Y^{h}\left(t_{0}\right)\right\|_{L^{2}\left(\Omega_{h}\right)}^{2} \leq 3 \sum_{k=1}^{N} \sum_{r=1}^{d}\left|\left\langle Y^{0, h}, \sum_{l=0}^{r-1} \Phi_{k}^{r-l, h} \frac{\left(-t_{0}\right)^{l}}{l !} \exp \left(-\lambda_{k}^{h} t_{0}\right)\right\rangle_{L^{2}\left(\Omega_{h}\right)}\right|^{2} \\
& +\left|\int_{0}^{t_{0}}\left\langle V_{d}^{h}(t),{ }^{t} \mathcal{D}_{\omega}^{h t} \mathrm{~B}^{h} \sum_{l=0}^{r-1} \Phi_{k}^{r-l, h} \frac{\left(t-t_{0}\right)^{l}}{l !} \exp \left(-\lambda_{k}^{h}\left(t_{0}-t\right)\right)\right\rangle_{L^{2}\left(\Omega_{h}\right)} d t\right|^{2} \\
& +\left(\frac{\Theta_{h}\|\gamma\|_{\infty}}{h} \frac{\left\|\phi_{k}^{h}\right\|_{L^{\infty}}}{(r-1) !}\right)^{2}\left|\int_{0}^{t_{0}} V_{b}^{h}(t)\left(t-t_{0}\right)^{r-1} \exp \left(-\lambda_{k}^{h}\left(t_{0}-t\right)\right) d t\right|^{2}
\end{aligned}
$$


SPECTRAL ANALYSIS OF DISCRETE ELLIPTIC OPERATORS AND APPLICATIONS 41

Thus,

$$
\begin{aligned}
\left\|Y^{h}\left(t_{0}\right)\right\|_{L^{2}\left(\Omega_{h}\right)}^{2} & \leq C\left(t_{0}, \beta, \gamma\right) \sum_{k=1}^{N} \sum_{r=1}^{d}\left|\left\langle Y^{0, h}, \Phi_{k}^{r, h}\right\rangle_{L^{2}\left(\Omega_{h}\right)}\right|^{2} \\
& +\left|\int_{0}^{t_{0}}\left\langle V_{d}^{h}(t),{ }^{t} \mathcal{D}_{\omega} \Phi_{k}^{1, h} \exp \left(-\lambda_{k}^{h}\left(t_{0}-t\right)\right)\right\rangle_{L^{2}\left(\Omega_{h}\right)} d t\right|^{2} \\
& +\frac{1}{h^{3}}\left|\int_{0}^{t_{0}} V_{b}^{h}(t) \exp \left(-\lambda_{k}^{h}\left(t_{0}-t\right)\right) d t\right|^{2} .
\end{aligned}
$$

In the last inequality we used that $\left\|\phi_{k}^{h}\right\|_{L^{\infty}}^{2} \leq \frac{\Theta_{h}}{h}\left\|\phi_{k}^{h}\right\|_{L^{2}\left(\Omega_{h}\right)}^{2} \leq \frac{\beta}{h}$.

$$
\left\|Y^{h}\left(t_{0}\right)\right\|_{L^{2}\left(\Omega_{h}\right)}^{2} \leq C\left[\left\|Y^{0, h}\right\|_{L^{2}\left(\Omega_{h}\right)}^{2}+\left\|V_{d}^{h}\right\|_{L^{2}\left(\Omega_{h}^{T}\right)}^{2} \sum_{k=1}^{N} \frac{1}{\lambda_{k}^{h}}+\frac{1}{h^{3}}\left\|V_{b}^{h}\right\|_{L^{2}(0, T)}^{2} \sum_{k=1}^{N} \frac{1}{\lambda_{k}^{h}}\right] .
$$

Finally, taking the square root and using the lower bounds for $\lambda_{k}^{h}$ given in Lemma 3.3 , we conclude that

$$
\left\|Y^{h}\left(t_{0}\right)\right\|_{L^{2}\left(\Omega_{h}\right)} \leq C\left[\left\|Y^{0, h}\right\|_{L^{2}\left(\Omega_{h}\right)}+\left\|V_{d}^{h}\right\|_{L^{2}\left(\Omega_{h}^{T}\right)}+\frac{1}{h^{3 / 2}}\left\|V_{b}^{h}\right\|_{L^{2}(0, T)}\right] .
$$

Now, we take advantage of the assumption (69) that implies the exponential decay of coefficients $\left\langle\Phi_{k}^{r, h}, Y^{h}(t)\right\rangle_{L^{2}\left(\Omega_{h}\right)}$ for $t \geq t_{0}$. Indeed, since both souce terms $V_{d}^{h}$ and $V_{b}^{h}$ are null after time $t_{0}$, the solution at time $T$ writes,

$$
Y^{h}(T)=\sum_{r=1}^{d} \sum_{k=K+1}^{N}\left\langle\Phi_{k}^{r, h}, Y^{h}\left(t_{0}\right)\right\rangle_{L^{2}\left(\Omega_{h}\right)} \exp \left(-\lambda_{k}^{h}\left(T-t_{0}\right)\right) \Phi_{k}^{r, h} .
$$

Thus,

$$
\left\|Y^{h}(T)\right\|_{L^{2}\left(\Omega_{h}\right)} \leq \exp \left(-\lambda_{K+1}^{h}\left(T-t_{0}\right)\right)\left\|Y^{h}\left(t_{0}\right)\right\|_{L^{2}\left(\Omega_{h}\right)},
$$

which gives the claim with estimate $(70)$.

Now we can state the main theorem of this section which applies in setting (S2).

THEOREM 5.4. Let $T>0$ and suppose that the mesh is uniform and that $q$ and $\gamma$ satisfy hypothesis $\left(\mathrm{H}_{0}\right)$. Let any function $\phi: \mathbb{R}_{+}^{*} \rightarrow \mathbb{R}_{+}^{*}$ such that

$$
\liminf _{h \rightarrow 0}\left[h^{2} \log (\phi(h))\right]>-8 \gamma_{\min } T .
$$

Then, system $\left(S_{d}^{h}\right)$ is $\phi(h)$-null controllable in time $T$.

Proof. The proof follows the same lines as the ones of Theorem 5.3 except that we only set to 0 a given portion of the Fourier modes of the solution.

From (71) we can find $t_{0} \in(0, T)$ and $\varepsilon \in(0,1)$ (depending only on $\phi$ ) such that

$$
\liminf _{h \rightarrow 0}\left[h^{2} \log (\phi(h))\right]>-8 \gamma_{\min }\left(T-t_{0}\right)(1-\varepsilon) .
$$

Let $k_{\max , \varepsilon}^{h}$ as in (29). We define $\tilde{\Lambda}_{\varepsilon}^{h}$ as follows

$$
\tilde{\Lambda}_{\varepsilon}^{h}:=\left\{\begin{array}{l}
\lambda_{k}^{h} \text { for } k \in \llbracket 1, k_{\text {max }, \varepsilon}^{h} \rrbracket \\
\lambda_{k_{\text {max }, \varepsilon}^{h}}^{h}+4 \gamma_{\min } k^{2} \text { for } k \geq k_{\max , \varepsilon}^{h}+1 .
\end{array}\right.
$$


Thanks to the gap estimate in Theorem 3.2, there exists a $\kappa_{\varepsilon}>0$ such that

$$
\tilde{\Lambda}_{\varepsilon}^{h} \in \mathcal{L}\left(\kappa_{\varepsilon}, \mathcal{N}\right), \quad \text { for any } h, \text { where } \mathcal{N} \text { is defined by }(63) .
$$

We apply Theorem 5.1 with $T$ replaced by $t_{0}$ and $\tau=t_{0} / 2$, in such a way that the biorthogonal family we obtain in $L^{2}\left(0, t_{0}\right)$ satisfies

$$
\left\|q_{j, l}^{\tilde{\Lambda}_{\varepsilon}^{h}}\right\|_{L^{2}\left(0, t_{0}\right)} \leq K_{t_{0}, \varepsilon} e^{\lambda_{l}^{h} t_{0} / 2}, \quad \forall l \in \llbracket 1, k_{\max , \varepsilon}^{h} \rrbracket, \forall j \in \llbracket 0, d-1 \rrbracket .
$$

We define now the following controls on $\left(0, t_{0}\right)$

$$
V_{b}^{h}(t)=\sum_{k=1}^{k_{\max , \varepsilon}^{h}} \sum_{r=1}^{d}(r-1) ! \frac{\left\langle Y^{0, h}, e^{-t_{0} \mathcal{L}^{h}} \Phi_{k}^{h, r}\right\rangle_{L^{2}\left(\Omega_{h}\right)}}{\gamma(1) \partial_{r} \phi_{k}^{h}} q_{r-1, k}^{\tilde{\Lambda}_{\varepsilon}^{h}}(t), \text { and } V_{d}^{h}(t)=0
$$

in the boundary control case and

$$
V_{d}^{h}(t)=\sum_{k=1}^{k_{\text {max }, \varepsilon}^{h}} \sum_{r=1}^{d}(r-1) ! \frac{-\left\langle Y^{0, h}, e^{-t_{0} \mathcal{L}^{h}} \Phi_{k}^{h, r}\right\rangle_{L^{2}\left(\Omega_{h}\right)}}{\left\|\phi_{k}^{h}\right\|_{L^{2}\left(\omega_{h}\right)}^{2}} q_{r-1, k}^{\tilde{\Lambda}_{\varepsilon}^{h}}(t) \phi_{k}^{h}, \text { and } V_{b}^{h}(t)=0,
$$

in the distributed control case. Using the above estimates on the biorthogonal family and the lower bounds on $\partial_{r} \phi_{k}^{h}$ and $\left\|\phi_{k}^{h}\right\|_{L^{2}\left(\omega_{h}\right)}$ given in Theorem 3.2, we obtain

$$
\left\{\begin{array}{l}
\left\|V_{d}^{h}\right\|_{L^{2}\left(\Omega_{h}^{t_{0}}\right)} \leq C_{\varepsilon, t_{0}}\left\|Y^{0, h}\right\|_{L^{2}\left(\Omega_{h}\right)}, \\
\left\|V_{b}^{h}\right\|_{L^{2}\left(0, t_{0}\right)} \leq C_{\varepsilon, t_{0}}\left\|Y^{0, h}\right\|_{L^{2}\left(\Omega_{h}\right)},
\end{array}\right.
$$

and moreover, by construction, we have

$$
\left\langle\Phi_{k}^{r, h}, Y^{h}\left(t_{0}\right)\right\rangle_{L^{2}\left(\Omega_{h}\right)}=0, \forall k \in \llbracket 1, k_{\max , \varepsilon}^{h} \rrbracket, \forall r \in \llbracket 1, d \rrbracket .
$$

Therefore, we can use Lemma 5.1 with $K=k_{\max , \varepsilon}^{h}$ to conlude that

$$
\begin{aligned}
\left\|Y^{h}(T)\right\|_{L^{2}\left(\Omega_{h}\right)} & \leq C_{t_{0}, \varepsilon}\left\|Y^{0, h}\right\|_{L^{2}\left(\Omega_{h}\right)}\left(1+\frac{1}{h^{3 / 2}}\right) e^{-\left(T-t_{0}\right) \lambda_{k_{\text {max }, \varepsilon}^{h}}^{h}+1} \\
& \leq C_{t_{0}, \varepsilon}\left\|Y^{0, h}\right\|_{L^{2}\left(\Omega_{h}\right)} \frac{1}{h^{3 / 2}} e^{-\left(T-t_{0}\right)(1-\varepsilon) \frac{4 \gamma_{\min }}{h^{2}}}
\end{aligned}
$$

By (72), for $h$ small enough, we conclude that

$$
\left\|Y^{h}(T)\right\|_{L^{2}\left(\Omega_{h}\right)}^{2} \leq C_{t_{0}, \varepsilon}\left\|Y^{0, h}\right\|_{L^{2}\left(\Omega_{h}\right)}^{2} \phi(h) .
$$

With the bounds (75), the claim follows.

We can now state an analogous theorem in the setting (S1).

Theorem 5.5. Let $T>0$. Suppose that $\gamma$ and $q$ satisfy $\left(\mathrm{H}_{2}\right)$. For any $\beta>0$, there exists $\alpha(q, \gamma, \beta)>0$, such that for any function $\phi: \mathbb{R}_{+}^{*} \rightarrow \mathbb{R}_{+}^{*}$ satisfying

$$
\liminf _{h \rightarrow 0}\left[h^{2 / 5} \log (\phi(h))\right]>-\alpha T,
$$

and any mesh family such that $\Theta_{h} \leq \beta$, the system $\left(S_{d}^{h}\right)$ is $\phi(h)$-null controllable at time $T$. 
Proof. This proof is similar to the proof of the previous theorem and the details are left to the reader. However, we precise below their main differences.

Let $\alpha>0$, as in the last estimate of Theorem 3.1 and define

$$
\tilde{\Lambda}^{h}:=\left\{\begin{array}{l}
\lambda_{k}^{h} \text { for } k \in \llbracket 1, \alpha N^{2 / 5}-1 \rrbracket, \\
\lambda_{k}^{h}+4 \gamma_{\min } k^{2} \text { for } k \geq \alpha N^{2 / 5} .
\end{array}\right.
$$

Thanks to the gap estimate of Theorem 3.1, there exists $\kappa>0$ such that

$$
\tilde{\Lambda}^{h} \in \mathcal{L}(\kappa, \mathcal{N}), \quad \text { for any } h .
$$

By constructing the same kind of controls as before, we get

$$
\left\langle\Phi_{k}^{r, h}, Y^{h}\left(t_{0}\right)\right\rangle_{L^{2}\left(\Omega_{h}\right)}=0, \forall k \in \llbracket 1, \alpha N^{2 / 5} \rrbracket, \forall r \in \llbracket 1, d \rrbracket,
$$

which, using Lemma 5.1 with $K=\alpha N^{2 / 5}$, leads to

$$
\left\|Y^{h}(T)\right\|_{L^{2}\left(\Omega_{h}\right)} \leq C_{t_{0}}\left\|Y^{0, h}\right\|_{L^{2}\left(\Omega_{h}\right)} \frac{1}{h^{3 / 2}} \exp \left(-\frac{\left(T-t_{0}\right) \alpha}{h^{2 / 5}}\right) .
$$

and the claim follows.

REMARK 5.6. The notion of $\phi(h)$-null controllability has been introduced here to remedy the fact that the gap property does not hold for the entire spectrum in the general case. Note that if one is able to prove, on a particular choice of $\gamma$ and $q$, that the gap property is valid for the whole spectrum, then the estimates of Section 3 allow to conclude that uniform null controllability holds.

\section{Remarks and further results.}

1. In [18] the authors consider the problem of null-controllability at the boundary for the semi-discretized in space linear beam equation with hinged boundary conditions and constant diffusion coefficient. They discretize the operator $\partial_{x x x x}$ with finite differences in $1 \mathrm{D}$ on a uniform mesh. The equation writes:

$$
\left\{\begin{array}{l}
\left(Y^{h}\right)^{\prime \prime}(t)+\left(\mathcal{A}^{h}\right)^{2} Y^{h}(t)=\mathcal{B}_{r}^{h} V_{b}^{h}(t), t \in(0, T) \\
Y^{h}(0)=Y^{h, 0}, \quad\left(Y^{h}\right)^{\prime}(0)=Y^{h, 1},
\end{array}\right.
$$

where $Y^{h, 0}$ and $Y^{h, 1}$ are vectors in $\mathbb{R}^{N}$. The corresponding adjoint system with final datum $Z_{T}^{h} \in \mathbb{R}^{2 N}$ writes

$$
\left\{\begin{array}{l}
\left(Z^{h}\right)^{\prime}(t)+L^{h} Z^{h}(t)=0 \\
Z^{h}(T)=Z_{T}^{h}
\end{array}\right.
$$

where $L^{h}=\left(\begin{array}{cc}0 & -\mathrm{I}^{h} \\ \left(\mathcal{A}^{h}\right)^{2} & 0\end{array}\right)$. The authors show, via the moments method, that uniform null controllability holds for this equation for some initial data whose high frequencies have been filtered out. As for parabolic problems, their proof makes use of explicit computations on the eigenelements $\left(\mu_{k}^{h}, \psi_{k}^{h}\right)_{1 \leq|k| \leq N}$ of the operator $L^{h}$.

Using the discrete spectral estimates obtained in Section 3 of the present paper, it is very likely that one can adapt the ideas of [18] to obtain similar results for more general second order elliptic operator $\mathcal{A}^{h}$ and, more importantly, for non uniform grids. 
2. We give here some additional information about the theorical results mentionned in Section 4, test case 4, where the mesh is uniform, $q=0$ and $\gamma$ is piecewise constant and takes its largest value on the right side of the domain : $\mathcal{O}:=] 0.4,1\left[\right.$. First, note that the equation $\mathcal{A}^{h} \phi_{k}^{h}=\lambda_{k}^{h} \phi_{k}^{h}$ defines a linear recurrence relation with constant coefficients for the indices $i$ such that $x_{i} \in \Omega \backslash \mathcal{O}$, since $\gamma$ is constant in this region. Using the homogenous Dirichlet condition at $x=0$, we can prove by explicit computations that $I_{l}^{h}(N) \leq \exp \left(-\frac{C_{1}}{h}\right)$ and $I_{1}^{h}(N) \leq \exp \left(-\frac{C_{1}}{h}\right)$, where $C_{1}$ depends only on $\gamma$. Next, we check that the computations done in Theorem 3.2 and Proposition 3.2 are still valid locally where $\gamma$ is constant and in particular in $\mathcal{O}$. Doing this, we can show that $I_{r}^{h}(N) \geq C_{2}$ and $I_{2}^{h}(N) \geq C_{2}\left|\omega^{2}\right|$, where $C_{2}$ only depends on $\gamma$.

3. In Remark 5.4 we stressed that, for the distributed control problem on a uniform mesh, the exponentially small lower bound of Theorem 3.1 was enough to give a uniform bound on $V_{d}^{h}$. In [8, Th 6.1], the authors show that a partial discrete Lebeau-Robbiano inequality holds for a discretization of a second order elliptic operator of the form $\mathcal{A}=-\partial_{x}\left(\gamma \partial_{x} \cdot\right)$, on regular families of nonuniform meshes (as the ones considered in Remark 1.6). Thus, considering a uniform discretization, they obtain that there exist $C>0$ and $\varepsilon>0$, both independant of $h$, such that for any eigenvalue $\lambda_{k}^{h}$ satisfying $\lambda_{k}^{h} \leq \varepsilon / h^{2}$, we have $\left\|\phi_{k}^{h}\right\|_{L^{2}\left(\omega_{h}\right)} \geq \frac{1}{C} \exp \left(-C \sqrt{\lambda_{k}^{h}}\right)$. This is the same lower bound as the one of Theorem 3.1, but only for a fraction of the spectrum, and only for regular grids and coefficients. Note also that the Lebeau-Robbiano inequalities do not give any information on the normal derivatives of the eigenfunctions.

4. The techniques used in this paper seem to be clearly restricted to the $1 \mathrm{D}$ situation. However, in a forthcoming paper [1], we manage to use those results (with a more precise estimate of the control cost in short time like $e^{C / T}$ ) in addition to discrete Lebeau-Robbiano techniques to obtain similar results for the boundary control of multi-dimensional coupled systems in Cartesian geometries.

Observe that the multi-dimensional situation has a more complex behavior since, contrary to the 1D case (see Remark 1.4 and the beginning of Section 5.3 ), it may happen that the semi-discrete problem is not even approximately controllable for a constant coefficient operator discretized on a uniform mesh. This is a consequence of the counter-example by O. Kavian reported in [21] that shows that it exists eigenfunctions of the 5-point 2D discrete Laplace operator that are localized along the diagonal of the domain. Therefore, the associated discrete normal derivative is zero on almost every point of the boundary of the domain.

Acknowledgments. We would like to thank the anonymous referees for their very careful reading of the paper that helped us to improve the presentation of our results.

\section{REFERENCES}

[1] D. Allonsius And F. Boyer, Boundary null-controllability of semi-discrete coupled parabolic systems in some multi-dimensional geometries, preprint, (2018).

[2] F. Ammar-Khodja, A. Benabdallah, M. González-Burgos, and L. de Teresa, The Kalman condition for the boundary controllability of coupled parabolic systems. Bounds on biorthogonal families to complex matrix exponentials, J. Math. Pures Appl. (9), 96 (2011), pp. 555-590. 
[3] F. Ammar Khodja, A. Benabdallah, M. González-Burgos, and L. de Teresa, Minimal time for the null controllability of parabolic systems: the effect of the condensation index of complex sequences, J. Funct. Anal., 267 (2014), pp. 2077-2151.

[4] F. Ammar Khodja, A. Benabdallah, M. González-Burgos, and L. de Teresa, New phenomena for the null controllability of parabolic systems: minimal time and geometrical dependence, J. Math. Anal. Appl., 444 (2016), pp. 1071-1113.

[5] S. Barbeiro, J. Fereira, And R. GrigoriefF, Supraconvergence of a finite difference scheme for solutions in $H^{s}(0, L)$, IMA Journal of Numerical Analysis, 25 (2005), pp. 797-811.

[6] D. BoffI, Finite element approximation of eigenvalue problems, Acta Numerica, 19 (2010), pp. 1-120.

[7] F. BOYER, On the penalised HUM approach and its applications to the numerical approximation of null-controls for parabolic problems, in CANUM 2012, 41e Congrès National d'Analyse Numérique, vol. 41 of ESAIM Proc., EDP Sci., Les Ulis, 2013, pp. 15-58.

[8] F. Boyer, F. Hubert, and J. L. Rousseau, Discrete carleman estimates for elliptic operators and uniform controllability of semi-discretized parabolic equations, Journal de Mathématiques Pures et Appliquées, 93 (2010), pp. 240-276.

[9] F. Boyer, F. Hubert, and J. L. Rousseau, Discrete carleman estimates for elliptic operators in arbitrary dimension and applications, SIAM Journal on Control and Optimization, 48 (2010), pp. 5357-5397.

[10] F. Boyer and J. L. Rousseau, Carleman estimates for semi-discrete parabolic operators and application to the controllability of semi-linear semi-discrete parabolic equations, Annales de l'Institut Henri Poincaré (C) Non Linear Analysis, 31 (2014), pp. 1035-1078.

[11] J.-M. Coron, Control and nonlinearity, vol. 136 of Mathematical Surveys and Monographs, American Mathematical Society, Providence, RI, 2007.

[12] S. Ervedoza, A. Marica, And E. Zuazua, Numerical meshes ensuring uniform observability of one-dimensional waves: construction and analysis, IMA Journal of Numerical Analysis, 36 (2015), pp. 503-542.

[13] H. O. FATTORINi AND D. L. Russell, Uniform bounds on biorthogonal functions for real exponentials with an application to the control theory of parabolic equations, Quart. Appl. Math., 32 (1974/75), pp. 45-69.

[14] R. Glowinski, J.-L. Lions, And J. He, Exact and approximate controllability for distributed parameter systems, vol. 117 of Encyclopedia of Mathematics and its Applications, Cambridge University Press, Cambridge, 2008. A numerical approach.

[15] S. LABBÉ AND E. TRÉlat, Uniform controllability of semidiscrete approximations of parabolic control systems, Systems \& Control Letters, 55 (2006), pp. 597-609.

[16] J. LAGNESE, Control of wave processes with distributed controls supported on a subregion, SIAM J. Control Optim., 21 (1983), pp. 68-85.

[17] A. Lopez And E. Zuazua, Some new results related to the null controllability of the 1-d heat equation, in Séminaire sur les Équations aux Dérivées Partielles, 1997-1998, École Polytech., Palaiseau, 1998, pp. Exp. No. VIII, 22.

[18] S. Micu, I. Rovenţa, and L. E. Temereancă, Approximation of the controls for the linear beam equation, Math. Control Signals Systems, 28 (2016), pp. Art. 12, 53.

[19] J. Pöschel and E. Trubowitz, Inverse spectral theory, vol. 130 of Pure and Applied Mathematics, Academic Press, Inc., Boston, MA, 1987.

[20] H. F. Weinberger, Lower bounds for higher eigenvalues by finite difference methods, Pacific J. Math., 8 (1958), pp. 339-368; erratum, 941.

[21] E. Zuazua, Propagation, observation, and control of waves approximated by finite difference methods, SIAM Rev., 47 (2005), pp. 197-243. 